\title{
A Batch-Authenticated and Key Agreement Framework for P2P-Based Online Social Networks
}

\author{
Lo-Yao Yeh, Member, IEEE, Yu-Lun Huang, Member, IEEE, Anthony D. Joseph, Member, IEEE, \\ Shiuhpyng Winston Shieh, Senior Member, IEEE, and Woei-Jiunn Tsaur, Member, IEEE
}

\begin{abstract}
Online social networks (OSNs) such as Facebook and MySpace are flourishing because more and more people are using OSNs to share their interests with friends. Because security and privacy issues on OSNs are major concerns, we propose a security framework for simultaneously authenticating multiple users to improve the efficiency and security of peer-to-peer (P2P)-based OSNs. In the proposed framework, three batch authentication protocols are proposed, adopting the one-way hash function, ElGamal proxy encryption, and certificates as the underlying cryptosystems. The hash-based authentication protocol requires lower computational cost and is suitable for resource-limited devices. The proxy-based protocol is based on asymmetric encryption and can be used to exchange more information among users. The certificate-based protocol guarantees nonrepudiation of transactions by signatures. Without a centralized authentication server, the proposed framework can therefore facilitate the extension of an OSN with batched verifications. In this paper, we formally prove that the proposed batch authentication protocols are secure against both passive adversaries and impersonator attacks, can offer implicit key authentication, and require fewer messages to authenticate multiple users. We also show that our protocols can meet important security requirements, including mutual authentication, reputation, community authenticity, nonrepudiation, and flexibility. With these effective security features, our framework is appropriate for use in P2P-based OSNs.
\end{abstract}

Index Terms-Authentication protocol, batch authentication, Online social networks (OSNs), Peer to peer (P2P).

Manuscript received May 20, 2011; revised October 14, 2011 and December 5, 2011; accepted February 2, 2012. Date of publication February 23, 2012; date of current version May 9, 2012. This work was supported in part by the Team for Research in Ubiquitous Secure Technology Center, University of California, Berkeley, the National Science Council under Grant NSC 100-2218E-492-026 and Grant NSC 100-2219-E-212-001, the Networked Communications Program, the Industrial Technology Research Institute, the Institute for Information Industry, the Chung Shan Institute of Science and Technology, Chunghwa Telecomm., Bureau of Investigation, D-Link, the International Collaboration for Advancing Security Technology, and the Taiwan Information Security Center, Ministry of Education, Taiwan. The review of this paper was coordinated by Prof. J. Misic.

L.-Y. Yeh is with the Network and Information Security Division, National Center for High-Performance Computing, Hsinchu 30076, Taiwan, and also with the Department of Information Management, National Chi Nan University, Nantou 545, Taiwan (e-mail: lyyeh@nchc.narl.org.tw).

Y.-L. Huang is with the Department of Electrical and Control Engineering, National Chiao-Tung University, Hsinchu 300, Taiwan.

A. D. Joseph is with the Department of Electrical Engineering and Computer Sciences, University of California at Berkeley, Berkeley, CA 94720 USA.

S. W. Shieh is with Department of Computer Science, National Chiao Tung University, Hsinchu 300, Taiwan.

W.-J. Tsaur (Corresponding author) is with the Department of Information Management, Da-Yeh University, Changhua 51591, Taiwan (e-mail: wjtsaur@mail.dyu.edu.tw).

Color versions of one or more of the figures in this paper are available online at http://ieeexplore.ieee.org.

Digital Object Identifier 10.1109/TVT.2012.2188821

\section{INTRODUCTION}

$\mathbf{O}$ NLINE social networks (OSNs) such as Facebook, MySpace, and Twitter are increasingly popular services. People can share information and pictures with old acquaintances, as well as relationships with friends. It is estimated that half a billion registered users interact with friends over OSNs [1], [2]. However, the weak authentication and registration process of current OSNs may lead to some security attacks [3]. With the rapid growth of OSNs, more valuable information is stored on OSNs. Hence, the privacy and security issues inherent to OSNs have attracted much attention.

Peer-to-peer ( $\mathrm{P} 2 \mathrm{P})$ technology is considered in the design of next-generation OSNs. As described in [6], a P2P-based OSN consists of the following three levels: 1) the social network level represents members and their relationships; 2) the application service level implements the P2P-based application infrastructure; and 3) the communication and transport level provides transport services over networks such as the Internet or mobile ad hoc networks. Relying on the cooperation between a number of independent parties who are also OSN users, a decentralized P2P architecture can be adopted with merits, including strong privacy protection, better scalability, and a lowered requirement for continuous Internet connectivity [1], [4]. Furthermore, a $\mathrm{P} 2 \mathrm{P}$ architecture can take advantage of real social networks and geographic proximity to support local services. ${ }^{1}$

P2P-based OSNs is a relatively new trend. Only a few studies [3], [5] have designed their security protocols based on the P2P architecture. In 2009, Buchegger et al. [5] examined the feasibility of P2P-based OSNs and advocated the use of encryption techniques to ensure privacy issues. In [6], the authors described a decentralized and privacy-preserving OSN application called Safebook, but no specific protocol was defined. Ge et al. [3] proposed a message delivery scheme (VisualSec) based on a fully distributed method that attempts to provide authentication and key generation services. However, identity authentication was not fully addressed, and only the out-ofband $(\mathrm{OOB})$ authentication method is mentioned in VisualSec. Another alternative is to integrate the existing authentication protocols from P2P networks into the P2P-based OSNs. In 2003, Shardul et al. [8] divided P2P users into several groups (called troupes). Taking zero knowledge and CLIQUES as bases, each troupe required a central computing authority for authentication. Lee and Kim [9] also proposed an adaptive

\footnotetext{
${ }^{1}$ This paper focuses only on the security issues of P2P-based OSNs and not other challenging problems of $\mathrm{P} 2 \mathrm{P}$ networks such as global search and content distribution [7].
} 
authentication protocol based on the reputation of $\mathrm{P} 2 \mathrm{P}$ systems. The reputation of each peer affects the type of certificates to be used in authenticating a user. In 2006, Nguyen [10] adopted identity-based cryptography and preshare keys to design a simplified P2P device authentication protocol. However, these existing protocols suffer from the following weaknesses.

1) Most of the current security protocols [1], [5], [6] for $\mathrm{P} 2 \mathrm{P}$-based OSNs lack specific procedures.

2) In current security protocols for P2P-based OSNs, each user has to be authenticated by OOB methods, which may impede the extension speed of social networks.

3) Most of the existing protocols support only one-to-one authentication.

4) The existing protocols do not consider the restrictions of underlying devices such as computing power and memory limitations.

This paper proposes a framework to take advantage of the $\mathrm{P} 2 \mathrm{P}$ architecture, including geographic proximity. Under the proposed framework, three batch authentication protocols are designed, using different cryptographic primitives, for different devices in P2P-based OSNs. The novel contributions of this paper are listed as follows.

- The proposed framework reduces the communication cost required for authenticating users.

- Due to their different security properties, the proposed protocols can be realized on a variety of devices such as personal digital assistants (PDAs), mobile phones, and laptops.

- By incorporating different trust levels, the proposed protocols allow a user with a high trust level to help authenticate other users and achieve the extensibility of a social network.

- The proposed protocols support a one-to-many authentication, which is the basis of batch authentication, to simultaneously authenticate multiple users. To the best of our knowledge, this paper is the first study that offers oneto-many batch authentication in P2P-based OSNs.

The proposed protocols are proved to be capable of mutually authenticating communication peers and remain secure against passive adversaries.

This paper is organized as follows. We briefly introduce the cryptographic background in Section II. Section III presents the assumptions and notations used in the proposed protocols. The details of the proposed protocols are described in Section IV. Then, we give the proof of security analysis and a performance comparison in Sections V-B and VI, respectively. Finally, this paper is concluded in Section VII.

\section{ElGamal Proxy ENCRYPTION}

By applying proxy encryption, a ciphertext $C$ that is encrypted by one user is transformed to $\hat{C}$, which is decrypted by another user. In 2007, Huang et al. [12] realized proxy encryption using an ElGamal cryptosystem. In the ElGamal cryptosystem, two public parameters $p$ and $q$ are shared by all users, where $p$ is a prime that is equal to $2 q+1$, and $g$ is the generator in $\mathbb{Z}_{p}^{*}$.
Then, a sender $U_{A}$ randomly chooses a secret key $x$ in $\mathbb{Z}_{p}^{*}$ and makes $\beta=g^{x}$ as his/her public key. $U_{A}$ generates two cipher values $\left(C_{1}, C_{2}\right)$, where $C_{1}=g^{r} \bmod p, C_{2_{A}}=\xi\left(\beta^{r}\right)=$ $\xi\left(\left(g^{x}\right)^{r}\right) \bmod p, r \in \mathbb{Z}_{q}^{*}$ is a randomly selected number, and $\xi$ represents the message. Assuming that the receiver $U_{B}$ possesses his/her secret key $y$ and the proxy $U_{P}$ holds the transformation key $(y-x)$, the sender $U_{A}$ sends the cipher value $\left(C_{1}, C_{2}\right)$ to $U_{P}$, and the proxy $U_{P}$ implements the following two tasks:

- transforms the cipher value $C_{2_{A}}$ into $C_{2_{P}}=C_{2_{A}} \cdot C_{1}^{(y-x)}$;

- sends $\left(C_{1}, C_{2_{P}}\right)$ to the receiver $U_{B}$.

Upon receipt of $\left(C_{1}, C_{2_{P}}\right), U_{B}$ decrypts the cipher with his/her secret key $y$ and derives the message $\xi$.

The proxy $U_{P}$ not only alters the cipher $\left(C_{1}, C_{2_{A}}\right)$ but also distributes a tailor-made component to $U_{B}$. When adopting such an encryption method to our protocols, a minor modification is required for authenticating multiple users in one verification procedure. In our protocols, the proxy distributes the tailormade parameters to each peer, and the authentication requester relies on these parameters to decrypt the cipher, cooperatively made by authenticating peers. If the message contents are received and verified, these peers are considered authenticated.

\section{BAtCH AUthenticAtion FrameWORK}

\section{A. Background}

In P2P-based OSNs, each user may act as a client or a server. Based on the uniqueness of such characteristics, we propose three novel protocols for authenticating P2P-based OSN users in batch. In the proposed protocols, a user can simultaneously authenticate several users through a trusted friend.

With regard to the authentication of the trusted friend, similar to [1], [5], and [6], the OOB method is assumed in our protocols. For example, a face-to-face preauthentication method [11], [13] through a location-limited channel can be used for negotiating a shared secret key between two friends. Once a user (e.g., $\left.U_{X}\right)$ is authenticated by another user $\left(U_{R}\right), U_{X}$ becomes a friend of $U_{R}$ and further helps $U_{R}$ to scale up his/her social network. Note that the proposed protocols are different from other group key management protocols [15] due to the following reasons.

1) Different goals. In conventional group key management protocols, a group is formed for a temporary purpose. The group is dismissed when that temporary purpose is served, and then, the group members lose relationships with other group members. Our social-based batch authentication protocols are designed for authenticating friends in OSNs. Once a group member is authenticated, he/she can help friends for another batch authentication. Such authentication protocols help extend the social network of a user.

2) Different behaviors. In most group key management protocols, group members are authenticated by the group leader "one by one." That is, $n$ authentication messages are required to authenticate $n$ group members. Then, these members share one common group key for the group communication. In our batch authentication 


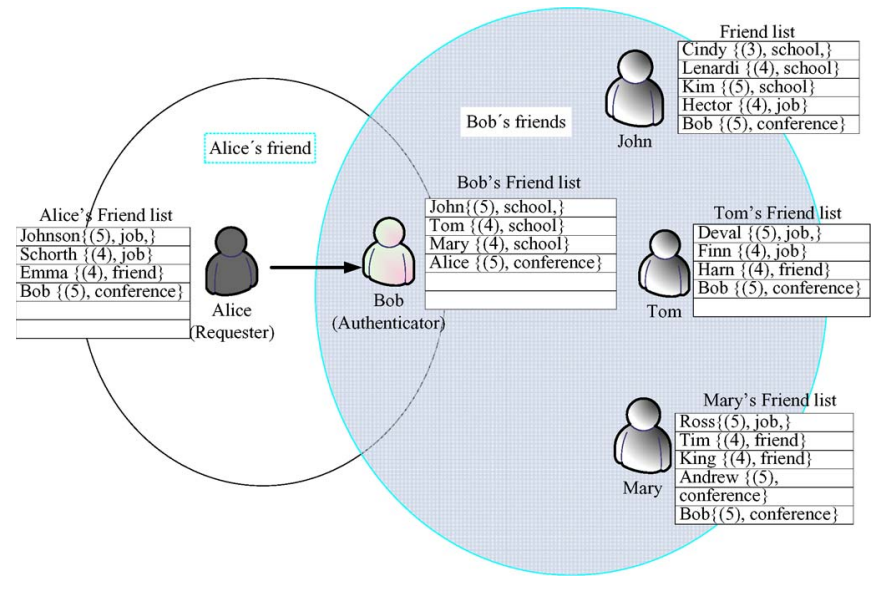

Fig. 1. Scenario of a batch authentication protocol.

protocols, users are simultaneously authenticated by the requester. That is, one authentication message is required to authenticate $n$ session peers. Then, the requester negotiates one secret key with each user instead of sharing one group key among all users.

In Fig. 1, for example, assume that Alice and Bob have performed a face-to-face preauthentication through a locationlimited channel (e.g., in a conference) in advance. They exchanged a secret shared key and shared friends lists, trust levels, and social relationships with each other after the preauthentication.

If Alice (who acts as the $U_{R}$ ) wants to communicate with users John, Tom, and Mary from Bob's friends list, she can ask Bob (who behaves as the $U_{A}$ ) to help the authentication. After successful authentication, Alice can exchange shared keys with John, Tom, and Mary and can add them into her friends list to broaden her social network.

\section{B. Requirements and Notations}

To support devices with different computing power, we propose three batch authentication protocols that adopt hash function, proxy encryption, and certification [16] as their underlying cryptography methods. The hash-based batch authentication protocol adopts a lightweight hash function and is suitable for resource-limited devices such as PDAs and cell phones. For devices with stronger computing power, the proxy-based batch authentication protocol can be applied to ensure confidentiality. The certificate-based batch authentication protocol can be used to protect sensitive and nonrepudiation transactions. These batch authentication protocols are explained in Section IV.

Considering the features of OSNs [1], [5], [6] and P2P architecture [9], we summarize the requirements of an authentication protocol for P2P-based OSNs as follows.

R1: Strong authentication. Mutual authentication should be guaranteed to guard against possible attacks.

R2: Reputation. Reputation ensures accountability in P2Pbased OSNs.

R3: Community authenticity. Each user should efficiently verify the source of messages. For example, a session key can be established with the use of keyed message authentication code.
R4: Nonrepudiation. Nonrepudiation must be guaranteed so that dispute for an invalid commercial transaction can be settled by a court.

R5: Flexibility. An authentication protocol should be adopted into any OSN, either web- or P2P-based networks.

R6: Batch authentication. Because a user may be far away from other users in a P2P network, authenticating multiple users in one transaction may reduce the communication cost.

R7: Comprehensive hardware support. An authentication protocol should support a variety of devices with different computing powers.

The notations used throughout this paper are listed in Table I.

\section{Sketch of the Proposed Batch Authentication}

The proposed batch authentication protocols, which are composed of three roles, a requester $U_{R}$, an authenticator $U_{A}$, and a user group $\hat{U}$, are operated based on the following assumptions.

1) The requester $U_{R}$ and authenticator $U_{A}$ have negotiated a shared key by face-to-face preauthentication through a location-limited channel [11], [13].

2) The authenticator $U_{A}$ is trusted by all his/her friends who are involved in the batch authentication.

3) If two users $U_{X}$ and $U_{Y}$ are friends, they have shared a secret key $K_{X Y}$.

In the proposed protocol, $U_{A}$ helps $U_{R}$ authenticate the user group $\hat{U}$, in which all users are friends of $U_{A}$. After successful authentication, $U_{R}$ establishes a shared key $K_{R i}$ with each user $U_{i}$ in the group $\left(U_{i} \in \hat{U}\right)$. We briefly explain our design concept by the following two cases. The detailed explanations of each message are introduced in Section IV.

In the first case, we introduce a user group with only one user $U_{1}\left(\hat{U}=\left\{U_{1}\right\}\right)$, as shown in Fig. 2(a). The message flow is given as follows.

1) $U_{R} \rightarrow U_{A}: A Q_{R, A}$.

2) $U_{A} \rightarrow U_{1}: C R_{A, 1}$.

3) $U_{1} \rightarrow U_{R}: C R_{1, R}$.

4) $U_{R} \rightarrow U_{1}: M R_{R, 1}$.

The requester $U_{R}$ initiates a request to the authenticator $U_{A}$. Then, $U_{A}$ helps contribute some parameters to $U_{R}$ and $U_{1}$ at Steps 2 and 3. Finally, $U_{R}$ replies a message $\left(M R_{R, 1}\right)$ at Step 4 for mutual authentication.

The second case scales up the user group to $n$ users $(\hat{U}=$ $\left\{U_{1}, U_{2}, \ldots, U_{n}\right\}$, and $\left.|\hat{U}|=n\right),{ }^{2}$ as shown in Fig. 2(b). The message flow is given as follows.

1) $U_{R} \rightarrow U_{A}: A Q_{R, A}$.

2) $U_{A} \rightarrow U_{1}: C R_{A, 1}$.

3) $U_{i-1} \rightarrow U_{i}: C R_{i-1, i}$, where $2 \leq i \leq|\hat{U}|$.

4) $U_{|\hat{U}|} \rightarrow U_{R}: C R_{|\hat{U}|, R}$.

5) $U_{R} \rightarrow U_{i}: M R_{R, i}$, where $1 \leq i \leq|\hat{U}|$.

\footnotetext{
${ }^{2}$ We assume that the $n$ users are online in the course of the batch verification or the batch authentication will fail. Note that the assumption can hold if $U_{A}$ can inform the users in $\hat{U}$ before the batch verification is executed.
} 
TABLE I

PARAMETERS AND NOTATIONS

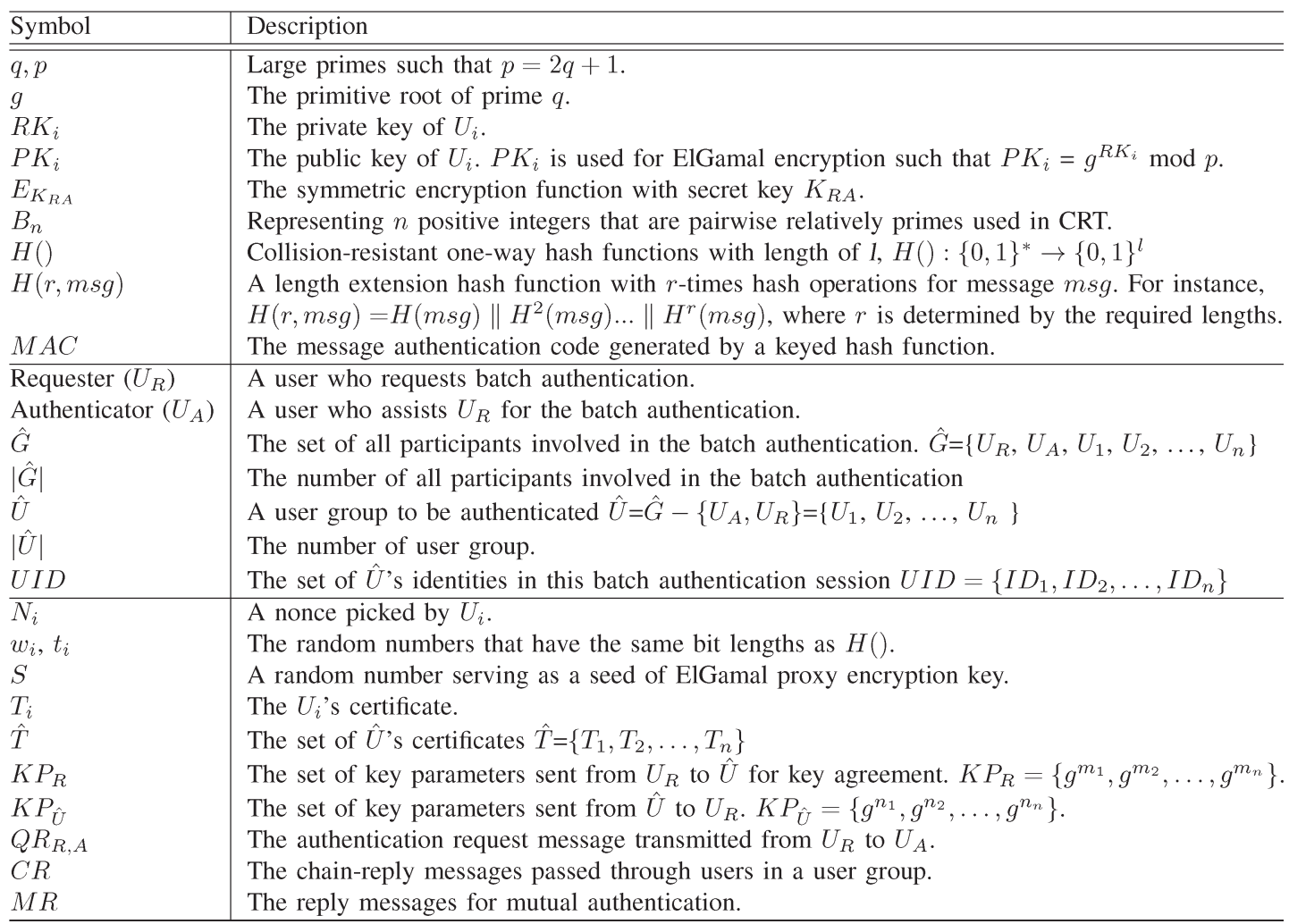
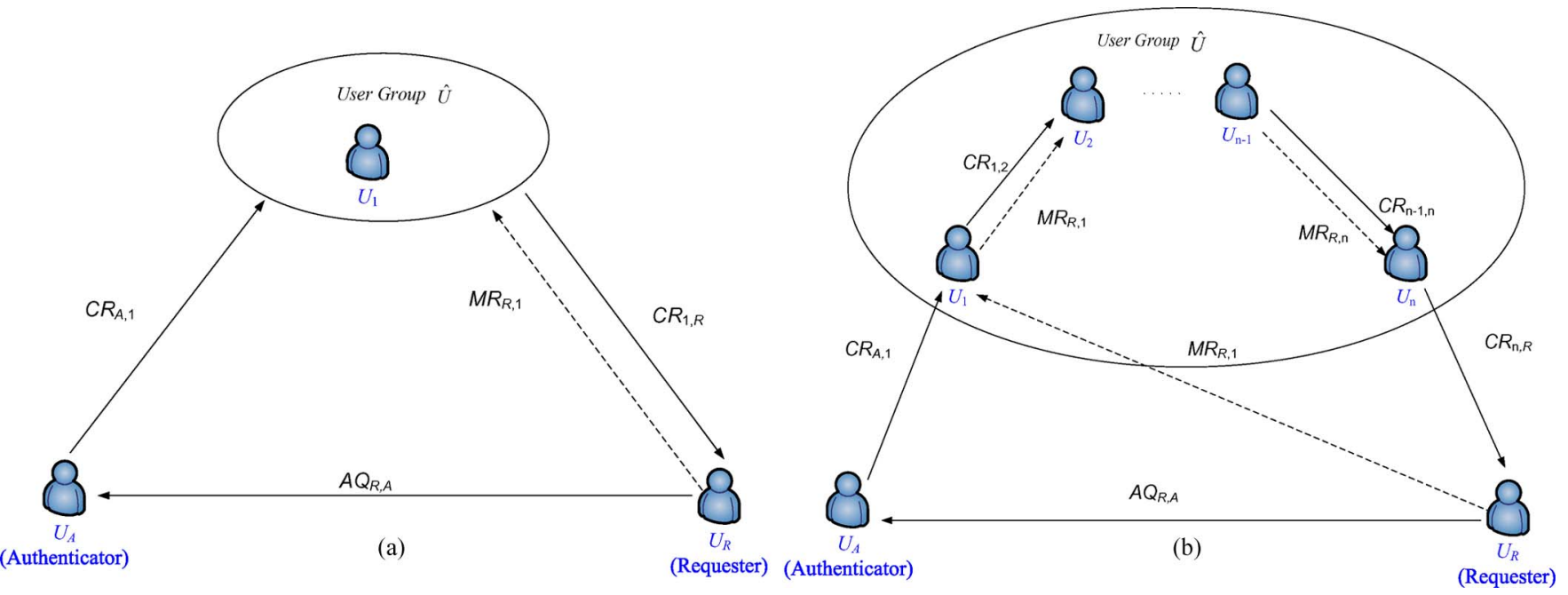

Fig. 2. Message flows of batch authentication for (a) only one member and (b) several members in case $\mathrm{n}=3$.

Similarly, $U_{R}$ sends a request to $U_{A}$. The authentication requests (chain reply $C R_{i, i+1}$ ) are then passed through $U_{1}, U_{2}, \ldots$ to $U_{n}$ at Steps 2 and $3^{*}$. At Step $4, U_{|\hat{U}|}$ sends back the chain reply to $U_{R}$. For mutual authentication, $U_{R}$ sends $M R_{R, i}$ to users $U_{i} \in \hat{U}$.

\section{Proposed Protocols}

This section explains the proposed batch protocols with different underlying cryptographic functions, including hash, proxy encryption, and certificates. Considering the security and performance issues, we recommend the proxy-based protocol as the default protocol in general situations.

\section{A. Hash-Based Protocol}

The hash-based batch authentication protocol, with the following message flow, is illustrated in Fig. 3.

1) $U_{R} \rightarrow U_{A}: A Q_{R, A}=\left\{I D_{R}, K_{R A} \oplus N_{R}, U I D \oplus H(r\right.$, $\left.\left.\left(N_{R}+1\right)\right), K P_{R} \oplus H\left(r,\left(N_{R}+2\right)\right), M A C_{R}\right\}$.

2) $U_{A} \rightarrow U_{1}: C R_{A, 1}=\left\{X, M A C_{A}\right\}$.

3) $U_{i-1} \rightarrow U_{i}: C R_{i-1, i}=\left\{M_{i}, X, K P_{\hat{U}}, M A C_{i}\right\}$, where $2 \leq i \leq|\hat{U}|$

4) $U_{|\hat{U}|} \rightarrow U_{R}: C R_{|\hat{U}|, R}=\left\{M_{|\hat{U}|}, X, K P_{\hat{U}}, M A C_{|\hat{U}|}\right\}$.

5) $U_{R} \rightarrow U_{i}: M R_{R, i}=\left\{Y, M A C_{R}^{\prime}\right\}$, where $1 \leq i \leq|\hat{U}|$. 


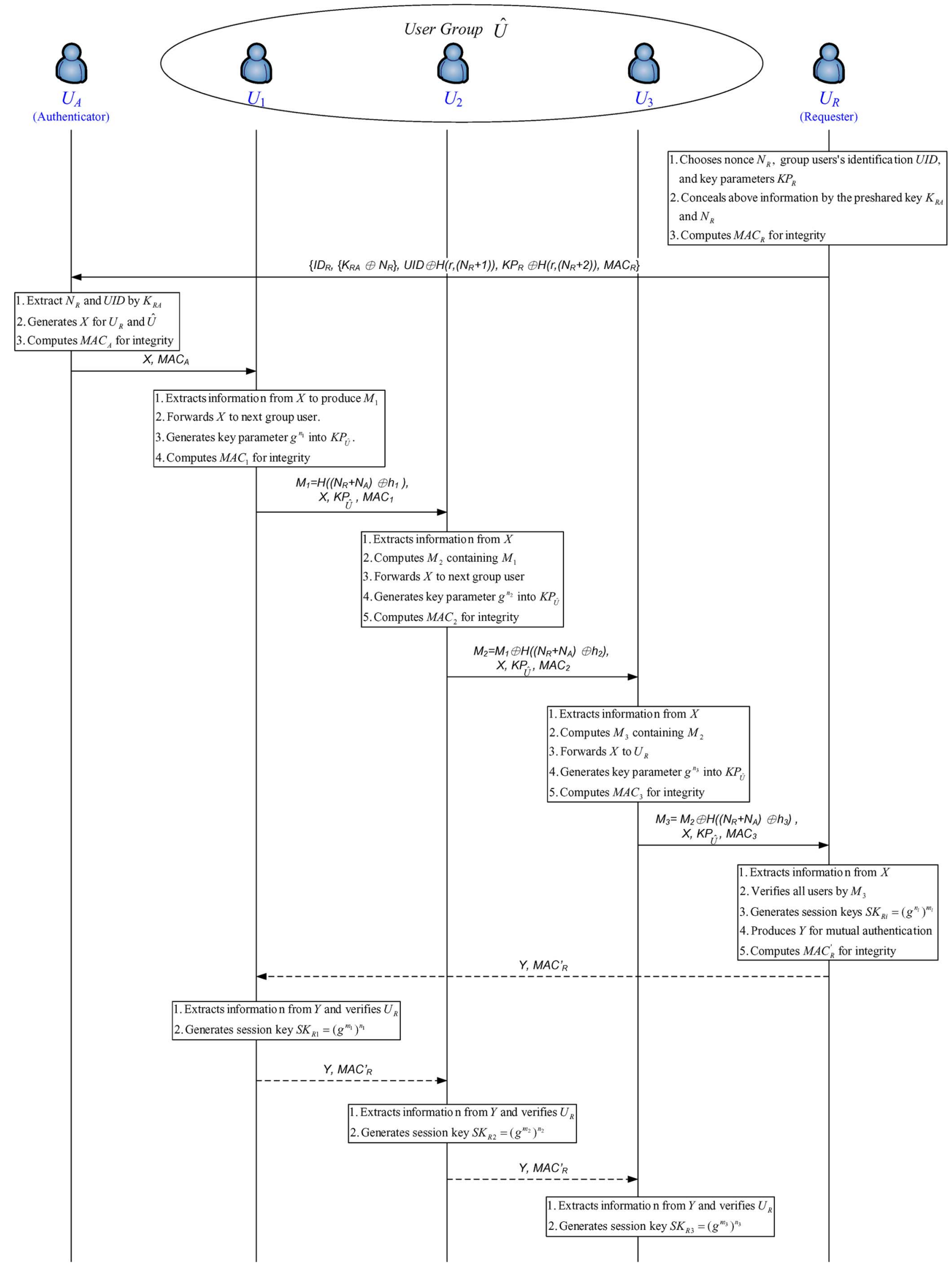

Fig. 3. Message flows of the hash-based batch authentication protocol. The illustration shows an example of a user group of size $3(|\hat{U}|=3)$. 


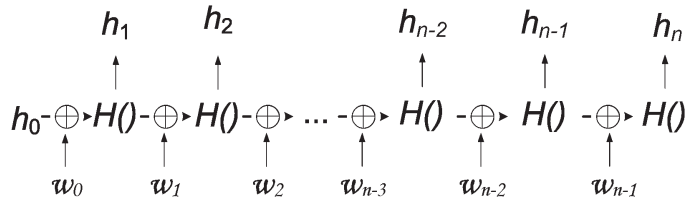

Fig. 4. Proposed one-way hash chain.

The hash-based batch authentication protocol is detailed as follows.

Step 1) $U_{R}$ sends $A Q_{R, A}$ to $U_{A} . A Q_{R, A}$ is composed of $U_{R}$ 's identification $\left(I D_{R}\right)$, a nonce $\left(N_{R}\right)$, the user group identification $\left(U I D=\left\{I D_{1}, I D_{2}, \ldots\right.\right.$, $\left.\left.I D_{|\hat{U}|}\right\}\right)$, and the parameters of key agreement $\left(K P_{R}=\left\{g^{m_{1}}, g^{m_{2}}, \ldots, g^{m_{\mid \hat{U}} \mid}\right\}\right.$, where $\left.m_{i} \in Z_{p}^{*}\right)$. The nonce is protected by a secret key $K_{R A}$ that is shared by $U_{R}$ and $U_{A}$. The group identification and key parameters are protected by the nonce. In addition, a message authentication code $M A C_{R}=$ $H\left(I D_{R},\left\{K_{R A} \oplus N_{R}\right\}, U I D \oplus H\left(r,\left(N_{R}+1\right)\right)\right.$, $\left.K P_{R} \oplus H\left(r,\left(N_{R}+2\right)\right),\left(N_{R}+3\right)\right)$ is attended to ensure the integrity of message.

Step 2) Upon the receipt of $A Q_{R, A}, U_{A}$ derives $N_{R}$ by performing $K_{R A} \oplus\left\{K_{R A} \oplus N_{R}\right\}$ and checks the validity of $M A C_{R}$. If $A Q_{R, A}$ is correct, the following steps are implemented.

- $U_{A}$ randomly generates an initial value $h_{0}$ and a sequence of random numbers $w_{i}$ (for $0 \leq i \leq$ $|\hat{U}|-1)$. Then, $U_{A}$ constructs and maintains a chain of one-way hash values $\left(h_{i}=H\left(h_{i-1} \oplus\right.\right.$ $\left.w_{i-1}\right)$ for $\left.1 \leq i \leq|\hat{U}|\right)$, as shown in Fig. 4.

- $U_{A}$ derives the user group identification $U I D$ and the key parameters $K P_{R}$ by $N_{R}$.

- $U_{A}$ computes $V_{0}$ for $U_{R}$, where

$V_{0}=\left\{I D_{A}, H\left(r,\left(K_{R A} \| t_{0}\right)\right)\right.$

$$
\left.\oplus\left(h_{0}\left\|H\left(K_{R A}\right)\right\| N_{A} \| \stackrel{|\hat{U}|-1}{\bigcup_{j=0}} w_{j}\right), t_{0}\right\} .
$$

Note that the unequal-bit-length problem can be solved by the specific length extension hash function $H(r, m s g)$ and $V_{0}$ should be regarded as a single element from the view of calculation. As mentioned in the previous section, $K_{R A}$ is the shared key between $U_{R}$ and $U_{A}, N_{A}$ and $t_{0}$ are random challenges from $U_{A}$, and $\cup_{j=0}^{|\hat{U}|-1} w_{j}$ is a concatenation of $w_{0}, w_{1}, \ldots, w_{|\hat{U}|-1}$.

- $U_{A}$ also computes $V_{i}$ for $U_{i} \in U,(1 \leq i \leq$ $|\hat{U}|)$, where

$$
\begin{aligned}
& V_{i}=\left\{I D_{A}, H\left(r,\left(K_{A i} \| t_{i}\right)\right)\right. \\
&\left.\oplus\left(h_{i}\left\|H\left(K_{A i}\right)\right\| N_{R}+N_{A} \| g^{m_{i}}\right), t_{i}\right\} .
\end{aligned}
$$

In $V_{i}(i \neq 0), g^{m_{i}}$ is used for negotiating session keys $K_{R i}$ between $U_{R}$ and $U_{i}$ in the end of the batch authentication.
- To eliminate the bandwidth requirements, we adopt the Chinese reminder theory (CRT) [17] to accommodate messages in a single message. Let $B_{0}, B_{1}, B_{2}, \ldots, B_{|\hat{U}|}$ be $|\hat{U}|+1$ positive integers that are pairwise relative primes and $A_{0}, A_{1}, A_{2}, \ldots, A_{|\hat{U}|}$ be the multiplicative inverses of $B_{0}, B_{1}, B_{2}, \ldots, B_{|\hat{U}|}$. $U_{A}$ computes a common solution $X$ for the following congruous equations:

$$
\begin{aligned}
& X \equiv V_{0} \bmod B_{0}\left(\text { for } U_{R}\right) \\
& X \equiv V_{i} \bmod B_{i}\left(\text { for } U_{i} \in U, 1 \leq i \leq|\hat{U}|\right) .
\end{aligned}
$$

By the CRT, we have $X=\left(\sum_{i=0}^{|\hat{U}|} L / B_{i} \times\right.$ $\left.V_{i} \times A_{i}\right) \bmod L$, where

$$
\begin{aligned}
& L=\prod_{i=0}^{|\hat{U}|} B_{i} \\
& A_{i} \times\left(L / B_{i}\right) \bmod B_{i} \equiv 1 .
\end{aligned}
$$

- $U_{A}$ calculates $M A C_{A}=H\left(X, N_{R}+N_{A}\right)$ and sends the chain reply $C R_{A, 1}=\{X$, $\left.M A C_{A}\right\}$ to the first user in the group $\left(U_{1}\right)$.

Step 3) After receiving $C R_{A, 1}=\left\{X, M A C_{A}\right\}$, the following steps are implemented.

- $U_{1}$ retrieves $V_{1}$ by calculating $X \bmod B_{1}$. Next, $\quad U_{1}$ obtains $H\left(r,\left(K_{A 1} \| t_{1}\right)\right) \oplus\left(h_{1} \|\right.$ $\left.H\left(K_{A 1}\right)\left\|N_{R}+N_{A}\right\| g^{m_{1}}\right)$ and $t_{1}$ from $V_{1} . U_{1}$ then uses the shared keys $K_{A 1}$ and $t_{1}$ to derive $h_{i}, H\left(K_{A 1}\right), N_{R}+N_{A}$, and $g^{m_{1}}$.

- The validity of $V_{1}$ and $C R_{A, 1}$ can be verified by $H\left(K_{A 1}\right)$ and $M A C_{A}$, respectively. The request is dropped when any invalidity is detected. Then, $U_{1}$ computes $M_{1}=H\left(\left(N_{R}+\right.\right.$ $\left.\left.N_{A}\right) \oplus h_{1}\right)$ and adds a key parameter $g^{n_{1}}$ to $K P_{\hat{U}}$.

- $U_{1}$ generates $M A C_{1}=H\left(M_{1}, X, K P_{\hat{U}}\right.$, $\left.\left(N_{R}+N_{A}\right)\right)$ and sends message $C R_{1,2}=\left\{M_{1}\right.$, $\left.X, K P_{\hat{U}}, M A C_{1}\right\}$ to the next group user $U_{2}$.

For $U_{i} \in U(2<i \leq|\hat{U}|)$, the following steps repeat until the chain reply passes through all group users.

- $U_{i} \quad$ gets $C R_{i-1, i}=\left\{M_{i-1}, X, K P_{\hat{U}}\right.$, $\left.M A C_{i-1}\right\}$ from $U_{i-1}$. $U_{i}$ extracts $V_{i}$ by $X \bmod B_{i}$. Similarly, $U_{i}$ can obtain $h_{i}$, $H\left(K_{A i}\right), \quad N_{R}+N_{A}, g^{m_{i}}$ from $V_{i}$ by the shared key $K_{A i}$ and random challenge $t_{i}$.

- The validity of $V_{i}$ and $C R_{i-1, i}$ can be verified by $H\left(K_{A i}\right)$ and $M A C_{i-1}$, respectively. When any invalidity is detected, the request is dropped, and $U_{i}$ reports the failure to $U_{A}$. Then, $U_{i}$ computes $M_{i}=M_{i-1} \oplus H\left(\left(N_{R}+N_{A}\right) \oplus\right.$ $\left.h_{i}\right)$ and adds a key parameter $g^{n_{i}}$ to $K P_{\hat{U}}$.

- $U_{i}$ generates $M A C_{i}=H\left(M_{i}, X, K P_{\hat{U}},\left(N_{R}+\right.\right.$ $\left.N_{A}\right)$ ) and sends $C R_{i, i+1}=\left\{M_{i}, X, K P_{\hat{U}}\right.$, $\left.M A C_{i}\right\}$ to the next group user $U_{i+1}$. 
Step 4) Upon the receipt of the last chain reply $C R_{|\hat{U}|, R}=$ $\left\{M_{|\hat{U}|}, X, K P_{\hat{U}}, M A C_{|\hat{U}|}\right\}$, the following steps are implemented.

- $U_{R}$ computes $X$ and $B_{0}$ and obtains $V_{0}$. With the shared key $K_{R A}$ and random challenge $t_{0}$, $U_{R}$ derives $h_{0}, H\left(K_{R A}\right), N_{A}$, and $\cup_{j=0}^{|\hat{U}|-1} w_{j}$ from $V_{0}$.

- Similarly, the authenticity of $V_{0}$ and $M A C_{|\hat{U}|}$ can be verified by $H\left(K_{R A}\right)$ and $M A C_{|\hat{U}|}$. If validated, $U_{R}$ derives $h_{i}(1 \leq i \leq|\hat{U}|)$ by $h_{0}$ and $\cup_{j=0}^{|\hat{U}|-1} w_{j}$.

- $U_{R}$ also computes $M_{|\hat{U}|}^{\prime}=H\left(\left(N_{R}+N_{A}\right) \oplus\right.$ $\left.h_{1}\right) \oplus H\left(\left(N_{R}+N_{A}\right) \oplus h_{2}\right) \oplus \cdots \oplus H\left(\left(N_{R}+\right.\right.$ $\left.\left.N_{A}\right) \oplus h_{|\hat{U}|}\right)$ and compares it with $M_{|\hat{U}|}$. If matched, the user group $\hat{U}$ is authenticated. Otherwise, at lease one of the users fails the authentication, and the session terminates.

- After the successful batch authentication, $U_{R}$ computes session keys $S K_{R i}=\left(g^{n_{i}}\right)^{m_{i}}$ for $U_{i}(1 \leq i \leq|\hat{U}|)$.

- For mutual authentication, $U_{R}$ calculates replies $\quad S_{i}=H\left(\left(N_{R}+N_{A}+1\right) \oplus h_{i}\right) \bmod$ $B_{i}$. Again, by applying the CRT [17], we can find a common solution for

$$
\begin{aligned}
Y \equiv S_{1} \bmod B_{1} \\
Y \equiv S_{2} \bmod B_{2} \\
\quad \vdots \\
Y \equiv S_{|\hat{U}|} \bmod B_{|\hat{U}|} .
\end{aligned}
$$

Then, $U_{R}$ generates $M A C_{R}^{\prime}=H\left(Y,\left(N_{R}+\right.\right.$ $\left.N_{A}\right)$ ) and sends $M R_{R, i}=\left\{Y, M A C_{R}^{\prime}\right\}$ to $U_{i}(1 \leq i \leq|\hat{U}|)$. In the case that $U_{R}$ cannot directly reach $U_{i}, U_{A}$ can be involved to help forward the messages.

Step 5) After receiving $M R_{R, i}$ from $U_{R}$ (or $U_{i-1}$ ), the following steps are implemented.

- $U_{i}$ first checks the validity of $M A C_{R}^{\prime}$.

- The session is dropped if $M A C_{R}^{\prime}$ fails the check; otherwise, $U_{i}$ computes $S_{i}=Y \bmod$ $B_{i}$ and checks the equality of $S_{i}^{\prime}$, where $S_{i}^{\prime}=$ $H\left(\left(N_{R}+N_{A}+1\right) \oplus h_{i}\right) \quad(1 \leq i \leq|\hat{U}|)$. If the equality holds, $U_{R}$ is authenticated; otherwise the session is terminated.

- After the successful batch authentication, $U_{i}$ computes the session key $S K_{R i}=\left(g^{m_{i}}\right)^{n_{i}}$. Subsequent communications between $U_{R}$ and $U_{i}$ can be protected by $S K_{R i}$.

\section{B. Proxy-Based Protocol}

By using the ElGamal proxy encryption scheme [12], the proxy-based protocol can carry trust levels [9] for entities that are involved in the batch authentication. In this protocol, we set two public parameters, $p$ and $g$, where $p$ is a prime of the form $2 q+1$, and $g$ is a generator in $\mathbb{Z}_{p}^{*}$. Similar to the hash- based protocol, the proxy-based batch authentication protocol comprises the following five messages, as illustrated in Fig. 5.

1) $U_{R} \rightarrow U_{A}: A Q_{R, A}=\left\{I D_{R},\left\{C 1, C 2_{R}\right\}, K_{R A} \oplus N_{R}\right.$, $\left.U I D \oplus H\left(r,\left(N_{R}+1\right)\right), M A C_{R}\right\}$.

2) $U_{A} \rightarrow U_{1}: C R_{A, 1}=\left\{C 1, C 2_{A}, X, M A C_{A}\right\}$.

3) $U_{i-1} \rightarrow U_{i}: C R_{i-1, i}=\left\{C 1, C 2_{i}, X, K P_{\hat{U}}, M A C_{i}\right\}$, where $2 \leq i \leq|\hat{U}|$.

4) $U_{|\hat{U}|} \rightarrow U_{R}: C R_{|\hat{U}|, R}=\left\{C 1, C 2_{|\hat{U}|}, X, K P_{\hat{U}}, M A C_{|\hat{U}|}\right\}$.

5) $U_{R} \rightarrow U_{i}: M R_{R, i}=\left\{C 2^{\prime}, M A C_{R}^{\prime}\right\}$, where $1 \leq i \leq|\hat{U}|$.

Step 1) The requester $U_{R}$ sets the shared key $K_{R A}$ as the seed of the ElGamal proxy encryption key and then starts the batch authentication protocol as follows.

- $U_{R}$ sends the authentication request $A Q_{R, A}=$ $\left\{I D_{R},\left\{C 1, C 2_{R}\right\}, K_{R A} \oplus N_{R}, U I D \oplus H(r\right.$, $\left.\left.\left(N_{R}+1\right)\right), M A C_{R}\right\}$ to $U_{A}$.

Step 2) Upon the receipt of $A Q_{R, A}$, the following steps are implemented.

- $U_{A}$ first derives $N_{R}$ by the shared key $K_{R A}$ and extracts the $U I D$ by $N_{R}$.

- Next, $U_{A}$ verifies $M A C_{R}$ and checks whether each $U_{i}$ 's trust level that is maintained by himself is higher than the predefined trust threshold. If one of the verifications fail, $U_{A}$ drops this session. Otherwise, $U_{A}$ computes $V_{0}$ for $U_{R}$ and $V_{i}$ for $U_{i} \in U$ as

$$
\begin{aligned}
& V_{0}=\left\{I D_{A}, E_{K_{R A}}\left(N_{A}, \sum_{j=1}^{|\hat{U}|} K_{A j}, H\left(K_{R A}\right)\right)\right\} \\
& V_{i}=\left\{I D_{A}, E_{K_{A i}}\left(K_{R A}+N_{R}+N_{A}+\sum_{\substack{j=1 \\
j \neq i}}^{|\hat{U}|} K_{A j}, H\left(K_{A i}\right)\right)\right\} .
\end{aligned}
$$

- Similarly, by applying the CRT [17], we can accommodate all replies in a single message as

$X \equiv V_{0} \bmod B_{0}\left(\right.$ for $\left.U_{R}\right)$

$X \equiv V_{1} \bmod B_{1}\left(\right.$ for $\left.U_{1}\right)$

$X \equiv V_{i} \bmod B_{i}\left(\right.$ for $\left.U_{i} \in U\right)$.

As mentioned in Section IV-A, by the CRT, we obtain $X=\left(\sum_{i=0}^{|\hat{U}|} L / B_{i} * V_{i} * A_{i}\right) \bmod L$.

- Based on the ElGamal proxy encryption scheme, $U_{A}$ calculates

$$
\begin{aligned}
C 2_{A} & =\left(C 2_{R} \times C 1^{N_{R}}\right) \bmod p \\
& =\left(\xi \beta^{r} \times g^{r\left(N_{R}\right)}\right) \bmod p \\
& =\left(\xi g^{\left(K_{R A}\right) r} \times g^{r\left(N_{R}\right)}\right) \bmod p \\
& =\left(\xi g^{r\left(K_{R A}+N_{R}\right)}\right) \bmod p
\end{aligned}
$$

- $U_{A}$ generates the message authentication code to protect the integrity of the message, where $M A C_{A}=H\left(C 1, C 2_{A}\right.$, $\left.X,\left(K_{R A}+N_{R}+N_{A}+\sum_{j=1}^{|\hat{U}|} K_{A j}\right)\right)$. 


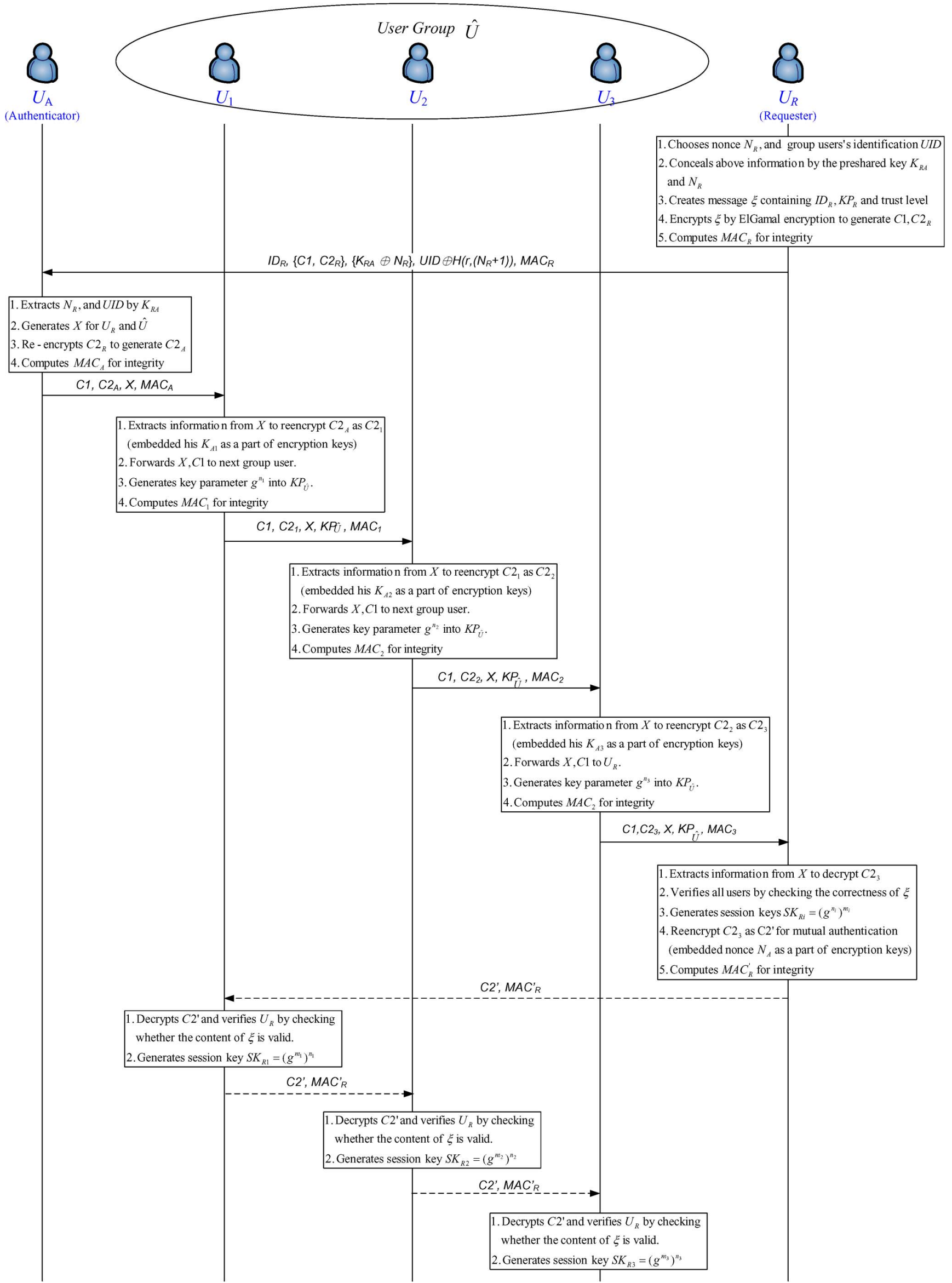

Fig. 5. Message flow of the proxy-based batch authentication protocol. The illustration shows an example of a user group of size $3(|\hat{U}|=3)$. 
- $U_{A}$ sends $C R_{A, 1}=\left\{C 1, C 2_{A}, X, M A C_{A}\right\}$ to $U_{1}$.

Step 3) After receiving $C R_{A, 1}$, the following steps are implemented.

- $U_{1}$ extracts $V_{1}=X \bmod B_{1}$ and decrypts $E_{K_{A i}}\left(K_{R A}+N_{R}+N_{A}+\sum_{j=2}^{|\hat{U}|} K_{A j}\right.$, $\left.H\left(K_{A 1}\right)\right)$ by $K_{A 1}$.

- $U_{1}$ verifies the integrity of $V_{1}$ and $C R_{A, 1}$ by checking $H\left(K_{A 1}\right)$ and $M A C_{A}$, respectively. The request is dropped when any invalidity is detected.

- $U_{1}$ calculates

$$
\begin{aligned}
C 2_{1} & =\left(C 2_{A} \times C 1^{\left(K_{A 1}\right)}\right) \bmod p \\
& =\left(\xi g^{r\left(K_{R A}+N_{R}\right)} \times g^{r\left(K_{A 1}\right)}\right) \bmod p . \\
& =\left(\xi g^{r\left(K_{R A}+N_{R}+K_{A 1}\right)}\right) \bmod p
\end{aligned}
$$

- $U_{1}$ selects the parameter of the session key $K P_{\hat{U}}=\left\{g^{n_{1}}\right\}$

- Because $K_{A 1}$ is shared with $U_{A}$ and $U_{1}$, only legitimate $U_{1}$ can decrypt $V_{1}$, add $K_{A 1}$ with $K_{R A}+N_{R}+N_{A}+\sum_{j=2}^{|\hat{U}|} K_{A j}$, and compute the message authentication code $M A C_{1}=$ $H\left(C 1, C 2_{1}, X, K P_{\hat{U}}, K_{R A}+N_{R}+N_{A}+\right.$ $\left.\sum_{j=1}^{|\hat{U}|} K_{A j}\right)$.

- $U_{1}$ sends $C R_{1,2}=\left\{C 1, C 2_{1}, X, K P_{\hat{U}}\right.$, $\left.M A C_{1}\right\}$ to $U_{2}$.

For $U_{i} \in U(2<i \leq|\hat{U}|)$, the following steps repeat until the chain reply passes through all group users.

- Upon the receipt of $C R_{i-1, i}, U_{i}$ derives $V_{i}=$ $X \bmod B_{i}$ and decrypts $E_{K_{A i}}\left(K_{R A}+N_{R}+\right.$ $\left.N_{A}+\sum_{\substack{j \neq i \\ j=1}}^{|\hat{U}|} K_{A j}, H\left(K_{A i}\right)\right)$ by $K_{A i}$.

- $U_{i}$ checks the validity of $H\left(K_{A i}\right)$ and $M A C_{i-1}$. The session is dropped if any invalidity is detected; otherwise, $U_{i}$ computes

$$
\begin{aligned}
C 2_{i} & =\left(C 2_{i-1} \times C 1^{\left(K_{A i}\right)}\right) \bmod p \\
& =\left(\xi g^{r\left(K_{R A}+N_{R}+\sum_{j=1}^{i-1} K_{A j}\right)} g^{r\left(K_{A i}\right)}\right) \bmod p \\
& \left.=\left(\xi g{ }^{r\left(K_{R A}+N_{R}+\sum_{j=1}^{i} K_{A j}\right.}\right)\right) \bmod p .
\end{aligned}
$$

- $U_{i}$ selects a parameter of session key $g^{n_{i}}$ and attaches it to $K P_{\hat{U}}$. Then, $U_{i}$ generates $M A C_{i}=H\left(C 1, C 2_{i}, X, K P_{\hat{U}}, K_{R A}+\right.$ $\left.N_{R}+N_{A}+\sum_{j=1}^{|\hat{U}|} K_{A j}\right)$.

- $U_{i}$ sends $C R_{i, i+1}=\left\{C 1, C 2_{i}, X, K P_{\hat{U}}\right.$, $\left.M A C_{i}\right\}$ to the next user $U_{i+1}$.
Step 4) After receiving $C R_{|\hat{U}|, R}$, the following steps are implemented.

- $U_{R}$ computes $V_{0}=X \bmod B_{0} \quad$ and decrypts $V_{0}$ by $K_{R A}$ to obtain $\left(N_{A}, \sum_{j=1}^{|U|} K_{A j}, H\left(K_{R A}\right)\right)$.

- $U_{R}$ checks the validity of $V_{0}$ by $H\left(K_{R A}\right)$ and $M A C_{|\hat{U}|}$. If valid, $U_{R}$ computes

$$
\begin{aligned}
\xi^{\prime}= & C 2_{|\hat{U}|} \times\left(C 1\left(K_{R A}+N_{R}+\sum_{j=1}^{|\hat{U}|} K_{A j}\right)\right. \\
= & \left(\xi g\left(K_{R A}+N_{R}+\sum_{j=1}^{|\hat{U}|} K_{A j}\right)\right) \\
& \times\left(g r\left(K_{R A}+N_{R}+\sum_{j=1}^{|\hat{U}|} K_{A j}\right)\right)^{-1} \bmod p .
\end{aligned}
$$

- If $\xi^{\prime}$ is identical to $\xi$, then all the group users $U_{i} \in \hat{U}$ are authenticated; otherwise, at least one group user fails the batch authentication.

- Once $\hat{U}$ is authenticated, $U_{R}$ can extract the key parameters of session key $g^{n_{i}}$ from $K P_{\hat{U}}$ and negotiate session keys with $U_{i} \in U$. The session keys can be obtained by $S K_{R i}=\left(g^{n_{i}}\right)^{m_{i}}$.

- For mutual authentication and key agreement, $U_{R}$ computes $C 2^{\prime}=$ $C 2_{|\hat{U}|} \times C 1^{N_{A}} \bmod p \quad$ and $\quad M A C_{R}^{\prime}=$ $H\left(C 2^{\prime}, K_{R A}+N_{R}+N_{A}+\sum_{j=1}^{|\hat{U}|} K_{A j}\right)$.

Then, the message $\left\{C 2^{\prime}, M A C_{R}^{\prime}\right\}$ is sent to $U_{i}(1 \leq i \leq|\hat{U}|)$. In the case that $U_{R}$ cannot directly reach $U_{i}, U_{A}$ can be involved to help forward the messages.

Step 5) After receiving $M R_{R, i}$ from $U_{R}$, the following steps are implemented.

- $U_{i}$ verifies the authenticity of $M A C_{R}^{\prime}$ and computes

$$
\begin{aligned}
& \xi^{\prime \prime}=C 2^{\prime} \times\left(C 1\left(K_{R A}+N_{R}+N_{A}+\sum_{j=1}^{|\hat{U}|} K_{A j}\right)\right)^{-1} \bmod p \\
& =\left(\xi g\left(K_{R A}+N_{R}+N_{A}+\sum_{j=1}^{|\hat{U}|} K_{A j}\right)\right) \\
& \times\left(g r\left(K_{R A}+N_{R}+N_{A}+\sum_{j=1}^{|\hat{\mathcal{O}}|} K_{A j}\right)\right)^{-1} \bmod p .
\end{aligned}
$$

- $U_{i}$ also checks whether $I D_{R}$ is included in $\xi^{\prime \prime}$. If yes, $U_{R}$ is authenticated. 


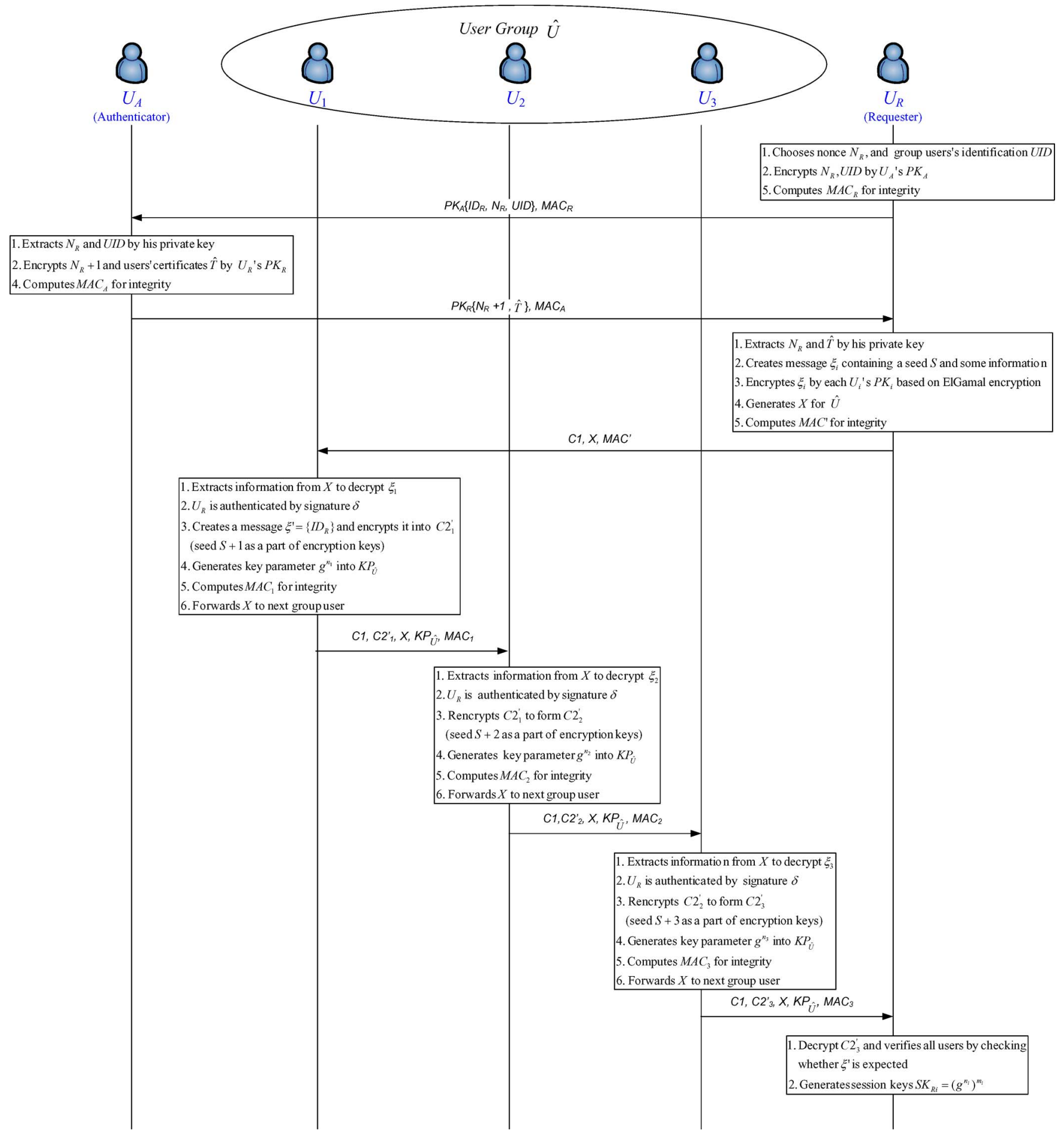

Fig. 6. Message flow of the certificate-based batch authentication protocol. The illustration shows an example of a user group of size $3(|\hat{U}|=3)$.

- Then, $U_{i}$ generates the session key $S K_{R i}=$ $\left(g^{m_{i}}\right)^{n_{i}}$ to protect the communication between $U_{R}$ and $U_{i}$.

\section{Certificate-Based Protocol}

The certificate-based protocol is proposed to guarantee the nonrepudiation of a transaction. In this protocol, we adopt the Shamir-Tauman online/offline signature [16] to enhance the security property. The authenticator $U_{A}$, behaving as a local trusted certificate authority, helps deliver and verify certificates for the group users $\left(U_{i} \in U\right)$. The message flow of the proposed certificate-based batch authentication protocol is summarized as follows (see Fig. 6).

1) $U_{R} \rightarrow U_{A}: A Q_{R, A}=\left\{P K_{A}\left\{I D_{R}, N_{R}, U I D\right\}, M A C_{R}\right\}$.

2) $U_{A} \rightarrow U_{R}: A R_{A, R}=\left\{P K_{R}\left\{N_{R}+1, \hat{T}\right\}, M A C_{A}\right\}$.

3) $U_{R} \rightarrow U_{1}: C R_{R, 1}=\left\{C 1, X, M A C_{A}\right\}$. 
4) $U_{i-1} \rightarrow U_{i}: C R_{i-1, i}=\left\{C 1, C 2_{i}^{\prime}, X, K P_{\hat{U}}, M A C_{i}\right\}$, where $2 \leq i \leq|\hat{U}|$

5) $U_{|\hat{U}|} \rightarrow U_{R}: C R_{|\hat{U}|, R}=\left\{C 1, C 2_{|\hat{U}|}^{\prime}, X, K P_{\hat{U}}, M A C_{|\hat{U}|}\right\}$.

The certificate-based protocol is described through the following steps.

Step 1) $U_{R}$ sends $A Q_{R, A}$ to $U_{A}$, where $A Q_{R, A}=$ $\left\{P K_{A}\left\{I D_{R}, N_{R}, U I D=\left\{I D_{1}, I D_{2}, \ldots, I D_{|\hat{U}|}\right\}\right\}\right.$, $\left.M A C_{R}\right\}$. In this message, the requester identity $I D_{R}$, nonce $N_{R}$, and user group identification $U I D$ are protected by $U_{A}$ 's public key $\left(P K_{A}\right)$. The message authentication code $M A C_{R}$ is derived by $H\left(P K_{A}\left\{I D_{R}, N_{R}, U I D\right\}, K_{R A}+N_{R}\right)$ and used to guarantee the validity of the message.

Step 2) When $U_{A}$ receives the $A Q_{R, A}$, the following steps are implemented.

- $U_{A}$ decrypts $A Q_{R, A}$ and obtains $I D_{R}, N_{R}$, and $U I D$.

- $U_{A}$ checks the validity of $M A C_{R}$. If illegal, $U_{A}$ drops this connection; otherwise, $U_{A}$ confirms each $U_{i}$ 's trust level and then encrypts $\left\{N_{R}+1, \hat{T}\right\}$ by $U_{R}$ 's public key $\left(P K_{R}\right)$, where $\hat{T}=\left\{T_{1}, T_{2}, \ldots, T_{|U|}\right\}$. If any $U_{i}$ 's trust level is lower than the predefined threshold, $U_{A}$ should note the information to $U_{R}$.

- $U_{A}$ derives $M A C_{A}=H\left(P K_{R}\left\{N_{R}+1, \hat{T}\right\}\right.$, $\left.K_{R A}+N_{R}\right)$ to protect the validity of the message.

- $U_{A}$ sends $A R_{A, R}$ to $U_{R}$, where $A R_{A, R}=$ $\left\{P K_{R}\left\{N_{R}+1, \hat{T}\right\}, M A C_{A}\right\}$.

Step 3) After obtaining $A R_{A, R}$, the following steps are implemented.

- $U_{R}$ checks the validity of the message by $M A C_{A}$ and $N_{R}+1$. If invalid, $U_{R}$ immediately drops the session; otherwise, $U_{R}$ decrypts the message by his/her private key $R K_{R}$ and obtains $\hat{T}$. Similar to the proxy-based batch authentication protocol, $U_{R}$ computes the following values:

1) $C 1=g^{r}$, where $r$ is a randomly selected secret parameter $r \in \mathbb{Z}_{q}^{*}$;

2) $V_{i}=C 2_{i}, \quad$ where $\quad C 2_{i}=\xi_{i}\left(P K_{i}\right)^{r}=$ $\xi_{i}\left(g^{R K_{i}}\right)^{r}$, and $\xi_{i}=\{$ trust level, $S, \delta=$ $\left.\operatorname{Sign}\left(R K_{R}, C 1\right), T_{R}, g^{m_{i}}\right\}$, where $S$ is a random number used as the seed of the ElGamal proxy encryption key, and $\delta$ is a signature generated by the Shamir-Tauman online/offline signature protocol [16].

- In addition, by adopting the CRT [17], we can merge all messages to $U_{i} \in U$ in a single message. Letting $B_{1}, B_{2}, \ldots, B_{|\hat{U}|}$ be positive integers that are pairwise relative primes and $V_{1}, V_{2}, \ldots, V_{|U|}$ be positive integers, we intend to obtain the solution for the following congruous equations:

$X \equiv V_{i} \bmod B_{i}\left(\right.$ for $\left.U_{i} \in U, 1 \leq i \leq|\hat{U}|\right)$.
We can derive the common solution $X$ for $X=\left(\sum_{i=1}^{|\hat{U}|} L / B_{i} \times V_{i} \times A_{i}\right) \bmod L$, where $1 \equiv A_{i} \times\left(L / B_{i}\right) \bmod B_{i}, \quad$ and $\quad\left\{V_{i}, B_{i} \mid i \in\right.$ $\{1, \ldots,|\hat{U}|\}\}$. Let $X=V_{i} \bmod B_{i}$ for $1 \leq i \leq|\hat{U}|$. Then, the common solution $X$ should be within the range of $[1, L-1]$, where $L=\prod_{i=1}^{|\hat{U}|} B_{i}$.

- $U_{R}$ generates $M A C_{R}=H(C 1, X, S)$ and sends the message $C R_{R, 1}=\{C 1, X$, $\left.M A C_{R}\right\}$ to $U_{1}$.

Step 4) Upon the receipt of $C R_{R, 1}$, the following steps are implemented.

- $U_{1}$ extracts $V_{1}=X \bmod B_{1}$ to obtain $C 2_{1}=$ $\xi_{1}\left(P K_{1}\right)^{r}=\xi_{1}\left(g^{R K_{1}}\right)^{r}$.

- $U_{1}$ decrypts $C 2_{1}$ by his/her own private key $R K_{1}$ and obtains $\xi_{1}$, where

$$
\begin{aligned}
C 2_{1}\left(C 1^{R K_{1}}\right)^{-1} \bmod p & =\xi_{1}\left(P K_{1}\right)^{r}\left(C 1^{R K_{1}}\right)^{-1} \bmod p \\
& =\xi_{1}\left(g^{R K_{1}}\right)^{r}\left(\left(g^{r}\right)^{R K_{1}}\right)^{-1} \bmod p \\
& =\xi_{1}=\left\{\text { trust level, } S, \delta, T_{R}, g^{m_{1}}\right\} .
\end{aligned}
$$

- $U_{1}$ derives $S$ from $\xi_{1}$ and checks the validity of $M A C_{R}$. The session is dropped if $M A C_{R}$ is invalidated; otherwise, $U_{1}$ continues to verify the signature $\delta$ that is signed by $U_{R} . U_{R}$ is authenticated if the signature is valid.

- If $U_{R}$ is authenticated, $U_{1}$ selects a random number $n_{1}$, generates the parameter of session key $g^{n_{1}}$, and adds the parameter to $K P_{\hat{U}}=$ $\left\{g^{n_{1}}\right\}$. Note that $U_{1}$ can now obtain the session key $S K_{R 1}=\left(g^{m_{1}}\right)^{n_{1}}$ and use it to protect the session.

- $U_{1}$ generates $C 2_{1}^{\prime}=\xi^{\prime}\left(P K_{R}\right)^{S+1}=$ $\xi^{\prime}\left(g^{R K_{R}}\right)^{S+1}$, where $\xi^{\prime}=\left\{I D_{R}\right\}$.

- $U_{1}$ appends the authentication message code $M A C_{1}=H\left(C 1, C 2_{1}^{\prime}, X, K P_{\hat{U}}, S\right)$ to the message $C R_{1,2}=\left\{C 1, C 2_{1}^{\prime}, X, K P_{\hat{U}}\right.$, $\left.M A C_{1}\right\}$ and sends the message to $U_{2}$.

For $U_{i} \in U(2<i \leq|\hat{U}|)$, the following steps repeat until the chain reply passes through all group users.

- After receiving the $C R_{i-1, i}=\left\{C 1, C 2_{i-1}^{\prime}\right.$, $\left.X, K P_{\hat{U}}, M A C_{i-1}\right\}$

1) $U_{i}$ derives $V_{i}=X \bmod B_{i}$ to obtain $C 2_{i}=\xi_{i}\left(P K_{i}\right)^{r}=\xi_{i}\left(g^{R K_{i}}\right)^{r}$.

2) $U_{i}$ decrypts $C 2_{i}$ by his/her private key $R K_{i}$ and checks the validity of $M A C_{i-1}$ and $\delta\left(U_{R}\right.$ 's signature).

3) If $U_{R}$ is legitimate, $U_{i}$ adds the parameter $g^{n_{i}}$ to $K P_{\hat{U}}$ and generates a session key $S K_{R i}=\left(g^{m_{i}}\right)^{n_{i}}$.

- After extracting $S$ from $\xi_{i}, U_{i}$ computes

$$
\begin{aligned}
C 2_{i}^{\prime} & =C 2_{i-1}^{\prime}\left(P K_{R}\right)^{(S+i)} \bmod p \\
& =\xi^{\prime}\left(g^{R K_{R}}\right)\left(\sum_{j=1}^{i-1}(S+j)\right)\left(g^{R K_{R}}\right)^{(S+i))} \bmod p \\
& =\xi^{\prime}\left(g^{\left.R K_{R}\right)}{ }^{\left(\sum_{j=1}^{i}(S+j)\right)} \bmod p .\right.
\end{aligned}
$$


- $U_{i}$ generates $M A C_{i}=H\left(C 1, C 2_{i}^{\prime}, X\right.$, $\left.K P_{\hat{U}}=\left\{g^{n_{1}}, \ldots, g^{n_{i}}\right\}, S\right)$.

- $U_{i}$ delivers the message $C R_{i, i+1}=\{C 1$, $\left.C 2_{i}^{\prime}, X, K P_{\hat{U}}, M A C_{i}\right\}$ to the next user $U_{i+1}$.

Step 5) After receiving $C R_{|\hat{U}|, R}, U_{R}$ checks $M A C_{|\hat{U}|}$ by $S$. If validated, then $U_{R}$ can authenticate all group users $U_{i} \in U$ as follows.

- $U_{R}$ computes $C 1^{\prime}=g^{\left(\sum_{j=1}^{|\hat{U}|}(S+j)\right)} \bmod p$.

- $U_{R}$ derives $\xi^{\prime}$ by computing

$$
\begin{aligned}
C 2_{|\hat{U}|}^{\prime}\left(C 1^{\prime R K_{R}}\right)^{-1} \bmod p \\
=\xi^{\prime}\left(g^{\left.R K_{R}\right)}\left(\sum_{j=1}^{|\hat{U}|}(S+j)\right)\right. \\
\quad \times\left(g\left(\sum_{j=1}^{|\hat{U}|}(S+j)\right) R K_{R}\right) \bmod p \\
=\xi^{\prime} .
\end{aligned}
$$

- $U_{R}$ checks the validity of $\xi^{\prime}=\left\{I D_{R}\right\}$. If so, $U_{i} \in \hat{U}$ are authenticated.

- $U_{R}$ then generates the session keys $S K_{R i}=$ $\left(g^{n_{i}}\right)^{m_{i}}$ to protect the sessions with group users $U_{i} \in U$.

Different from the other two protocols, in the certificatebased protocol, $U_{R}$ knows whether $U_{i}$ is authenticated by checking the value of $\sum_{j=1}^{|\hat{U}|}(S+j)$. In a group of three users, for example, $U_{R}$ obtains $\sum_{j=1}^{3}(S+j)=3 S+6$ if all group users are authenticated. Therefore, if a smaller value, for example, $2 S+4$, is received, it is implied that some user $\left(U_{2}\right)$ has failed the authentication.

\section{Correctness And Security Analysis}

\section{A. Correctness Analysis}

In this section, we demonstrate the formula correctness of our protocols.

1) Hash-Based Protocol:

- User group authentication. As mentioned in Section IV-A, Step 3, each $U_{i}$ computes $M_{i}=M_{i-1} \oplus H\left(\left(N_{R}+N_{A}\right) \oplus\right.$ $\left.h_{i}\right)$. If $1 \leq i \leq|\hat{U}|$. Then, $M_{|\hat{U}|}=H\left(\left(N_{R}+N_{A}\right) \oplus h_{1}\right) \oplus$ $H\left(\left(N_{R}+N_{A}\right) \oplus h_{2}\right) \oplus \cdots \oplus H\left(\left(N_{R}+N_{A}\right) \oplus h_{|\hat{U}|}\right.$. Therefore, in Section IV-A, Step 4, $U_{R}$ will check whether $M_{|\hat{U}|}^{\prime} \stackrel{?}{=} M_{|\hat{U}|}$ holds, which is verified as follows:

$$
\begin{aligned}
M_{|\hat{U}|}^{\prime}= & H\left(\left(N_{R}+N_{A}\right) \oplus h_{1}\right) \oplus H\left(\left(N_{R}+N_{A}\right) \oplus h_{2}\right) \\
& \oplus \ldots \oplus H\left(\left(N_{R}+N_{A}\right) \oplus h_{|\hat{U}|}\right) \\
= & M_{|\hat{U}|} .
\end{aligned}
$$

- Requester authentication. In Section IV-A, Step $4, U_{R}$ computes $S_{i}=H\left(\left(N_{R}+N_{A}+1\right) \oplus h_{i}\right)$ for each $U_{i}$. As a result, in Step 5, each $U_{i}$ can authenticate $U_{R}$ by checking whether $S_{i}^{\prime} \stackrel{?}{=} S_{i}$ holds as follows:

$$
S_{i}^{\prime}=H\left(\left(N_{R}+N_{A}+1\right) \oplus h_{i}\right)=S_{i} .
$$

\section{2) Proxy-Based Protocol:}

- User group authentication. In Section IV-B Step 3, each $U_{i}$ contributes its own key $K_{A i}$ into $C 2_{i}$. If $1 \leq i \leq|\hat{U}|$, then $C 2_{i}$ evolves into $C 2_{|\hat{U}|}$ as follows:

$$
\begin{aligned}
C 2_{i} & =\left(C 2_{A} \times C 1^{\left(K_{A i}\right)}\right) \text { for } 1 \leq i \leq|\hat{U}| \\
& =\xi g^{r\left(K_{R A}+N_{R}\right)} \times g^{r\left(K_{A 1}\right)} \times g^{r\left(K_{A 2}\right)} \times \cdots \times g^{r\left(K_{A|\hat{U}|}\right)} \\
& =\xi g^{r\left(K_{R A}+N_{R}\right)} \times g^{r\left(K_{A 1}\right)+r\left(K_{A 2}\right)+\cdots+r\left(K_{A|\hat{U}|}\right)} \\
& \quad{ }^{r}\left(K_{R A}+N_{R}+\sum_{j=1}^{|\hat{U}|} K_{A j}\right) \\
& =C 2_{|\hat{U}|} .
\end{aligned}
$$

As a result, in Section IV-B, Step 4, $U_{R}$ will authenticate the group users by checking whether $\xi^{\prime} \stackrel{?}{=} \xi$ holds, which follows the ElGamal proxy decryption and is verified as follows:

$\xi^{\prime}=C 2_{|\hat{U}|} \times\left(C 1^{\left(K_{R A}+N_{R}+\sum_{j=1}^{|\hat{U}|} K_{A j}\right)}\right)^{-1}$

$=\xi g^{r\left(K_{R A}+N_{R}+\sum_{j=1}^{|\hat{U}|} K_{A j}\right)} \times\left(g\left(K_{R A}+N_{R}+\sum_{j=1}^{|\hat{U}|} K_{A j}\right)\right)^{-1}$

$=\xi \bmod p$.

- Requester authentication. In Section IV-B, Step 4, $U_{R}$ embeds its $N_{A}$ into $C 2_{|\hat{U}|}$ to form $C 2^{\prime}$ as follows:

$$
\begin{aligned}
C 2^{\prime} & =C 2_{|\hat{U}|} \times C 1^{N_{A}} \\
& =\xi g{ }^{r\left(K_{R A}+N_{R}+\sum_{j=1}^{|\hat{U}|} K_{A j}\right)} \times g^{r\left(N_{A}\right)} \\
& =\xi g
\end{aligned}
$$

Therefore, in Section IV-B, Step 5, each $U_{i}$ also verifies the validity of $U_{R}$ by checking whether the form and 
content of $\xi^{\prime \prime}$ are correct. The decryption formula of $\xi^{\prime \prime}$ is shown as follows:

$$
\begin{aligned}
\xi^{\prime \prime}= & C 2^{\prime}, \times\left(C 1\left(K_{R A}+N_{R}+N_{A}+\sum_{j=1}^{|\hat{U}|} K_{A j}\right)\right)^{-1} \\
= & \xi g\left(K_{R A}+N_{R}+\sum_{j=1}^{|\hat{U}|} K_{A j}\right) \\
& \times\left(g\left(K_{R A}+N_{R}+N_{A}+\sum_{j=1}^{|\hat{U}|} K_{A j}\right)\right)^{-1} \\
= & \xi \bmod p .
\end{aligned}
$$

\section{3) Certificate-Based Protocol:}

- User group authentication. In Section IV-C Step 4, $U_{1}$ first generates $C 2_{1}^{\prime}=\xi^{\prime}\left(P K_{R}\right)^{S+1}=\xi^{\prime}\left(g^{R K_{R}}\right)^{S+1}$, and then, each $U_{i}$ for $2<i \leq|\hat{U}|$ contributes their $(S+i)$ into $C 2_{i}^{\prime}$. In the end, $C 2_{|\hat{U}|}^{\prime}$ is formed as follows:

$$
\begin{aligned}
C 2_{|\hat{U}|}^{\prime}= & C 2_{1}^{\prime} \times\left(P K_{R}\right)^{(S+2)} \times\left(P K_{R}\right)^{(S+3)} \\
& \left.\times \cdots \times\left(P K_{R}\right)^{(S+|\hat{U}|}\right) \bmod p \\
= & \xi^{\prime}\left(g^{R K_{R}}\right)^{(S+1)} \times\left(g^{R K_{R}}\right)^{(S+2)} \\
& \times \cdots \times\left(g^{R K_{R}}\right)^{(S+|\hat{U}|)} \bmod p \\
= & \xi^{\prime}\left(g^{R K_{R}}\right)^{\left(\sum_{j=1}^{|\hat{U}|}(S+j)\right)} \bmod p .
\end{aligned}
$$

Finally, in Section IV-C, Step 5, $U_{R}$ computes $C 1^{\prime}=$ $g^{\left(\sum_{j=1}^{|\hat{U}|}(S+j)\right)}$ for decryption, which follows the procedure for the ElGamal proxy cryptography. After obtaining $\xi^{\prime}, U_{R}$ can confirm whether the content of $\xi^{\prime}$ is legitimate. The correctness of the decryption is presented as follows:

$$
\begin{aligned}
C 2_{|\hat{U}|}^{\prime} & =C 2_{|\hat{U}|}^{\prime}\left(C 1^{\prime R K_{R}}\right)^{-1} \bmod p \\
& \left.=\xi^{\prime}\left(g^{R K_{R}}\right)\left(\sum_{j=1}^{|\hat{U}|}(S+j)\right)\left(g^{(\hat{U} \mid}(S+j)\right) R K_{R}\right)^{-1} \bmod p \\
& =\xi^{\prime} .
\end{aligned}
$$

- Requester authentication. Because the requester authentication is achieved by the verification of $U_{R}$ 's signature, the correctness of signature verification is referenced by the Shamir-Tauman online/offline signature [16].

\section{B. Security Analysis}

This section gives security proofs and analyzes the proposed protocols. Due to space limitations, we briefly introduce the security model and follow the concept of formal security proofs to reduce our protocols to well-known hard problems. ${ }^{3} \mathrm{Sim}$ ilar to [15], we prove the security of proxy- and certificatebased protocols based on the decision Diffie-Hellman (DDH) assumption [19], [20]. Then, we prove that the hash-based batch authentication protocol is secure against passive adversaries, impersonators, and implicit key authentication attacks by applying the concept of the random-oracle model [18].

1) Security Model and Concepts: In 1993, Bellare and Rogaway [18] proposed a theoretical security proof for an authentication and key agreement protocol, called the BR93-Model. In our security analyses, we take the BR93-Model as a foundation for defining the security analysis concept.

Protocol Participants: $\prod_{U_{*}, R}^{i}$ denotes the user oracle, which plays the role $U_{*}$ of interacting with $R$ in the $i$ th session, and $\prod_{R, U_{*}}^{j}$ denotes the server oracle, which plays the role $R$ of interacting with $U_{*}$ in the $j$ th session. Note that, in our scheme, the server is regarded as the combination of the authenticator and the requester. Let $\mathcal{F}$ be the proposed authentication protocols. During the execution of $\mathcal{F}$, an adversary $E$ who is a probabilistic polynomial-time Turing machine can control the entire network and obtain transmitted data in previous processes. The predefined oracle queries are listed as follows.

- Execute $\left(\prod_{U_{*}, R}^{i}, \prod_{R, U_{*}}^{j}\right)$. The query models all kinds of passive attacks, where a passive adversary can eavesdrop on all transmitted data between $\prod_{U_{*}, R}^{i}$ and $\prod_{R, U_{*}}^{j}$ in the protocol $\mathcal{F}$.

- Send $\left(\prod_{U_{*}, R}^{i}, \xi\right)$. The query models an active attack, where an adversary sends a message $\xi$ to $\prod_{U_{*}, R}^{i}$. The adversary can get the response message according to the sending message $\xi$ in the protocol $\mathcal{F}$. An adversary can initiate a session by setting $\xi=\lambda$.

- Send $\left(\prod_{R, U_{*}}^{j}, \xi\right)$. The query also models active attacks, where an adversary sends a message $\xi$ to $\prod_{R, U_{*}}^{j}$. The adversary can get the response message according to the sending message $\xi$ in the protocol $\mathcal{F}$.

- Reveal $\left(\prod_{U_{*}, R}^{i}\right)$. This query models the exposure of the old session key of instance $i$.

- Hash(). This query allows an adversary to ask the hash function value, and the challenger (or the simulator) answers all Hash() queries at random, just like real oracles would.

- Test $\left(\prod_{U_{*}, R}^{i}\right)$. If $\prod_{U_{*}, R}^{i}$ accepts and shares a session key with the partner oracle $\prod_{R, U_{*}}^{j}$, the adversary $E$ can launch the query to distinguish a real session key from a random string. The query models the adversary's queries of the test oracle. Depending on a random coin bit, the adversary $E$ is given the real session key or a randomly chosen string.

Security in the model is defined using the game $\mathcal{G}$, which is played between the adversary $E$ and a collection of oracles

\footnotetext{
${ }^{3}$ More specifically, we will not claim that our analysis is a formal security proof, because the details of query operations and probability calculation are
} omitted. See [29], [30] for a more formal proof. 
$\prod_{U_{*}, R}^{i} / \prod_{R, U_{*}}^{j}$. The adversary $E$ runs the game simulation $\mathcal{G}$ with the following setting.

Step 1) The adversary $E$ can perform Execute, Send, Reveal, Hash, and Test queries in the simulation.

Step 2) At any moment during $\mathcal{G}, E$ can choose a fresh session and send a Test query to the fresh oracle that is associated with the test session. Depending on a randomly chosen bit $\beta \in\{0,1\}$, the challenger returns either a real session key or a randomly chosen string.

Step 3) $E$ continues issuing the aforementioned Execute, Send, Reveal, and Hash queries.

Step 4) Finally, $E$ terminates the game simulation and outputs its guess bit $\beta^{\prime}$.

As we have done, we define the E's guessing advantage as

$$
\operatorname{Adv}^{E}(k)=\left|\operatorname{Pr}\left[\beta=\beta^{\prime}\right]-\frac{1}{2}\right|
$$

where $k$ is the security parameter.

Security Concept: The aforementioned queries represent the abilities of an adversary in a real environment. The purpose of the adversary is to either pass the authentication procedure or distinguish the session key from a random string with a nonnegligible probability. Our security analysis follows the concept that if an adversary was able to be authenticated or to distinguish the session key, the adversary could solve some well-known computationally hard problems.

2) Security Proof by the DDH Assumption: In the proxy- and certificate-based protocols, $U_{R}$ adopts the Shamir-Tauman online/offline signature protocol [16] to generate $\delta=\operatorname{Sign}\left(R K_{R}, C 1\right)$. Therefore, we prove the security of proxy- and certificate-based batch authentication protocols by the DDH assumption [19], [20].

Assumption 1-DDH Problem: There are primes $p$ and $q$ such that $p=2 q+1$ and a generator $g \in \mathbb{Z}_{p}^{*}$ with order $q$ for the subgroup $G_{q}$, where $G_{q}$ is a subgroup of quadratic residues in $\mathbb{Z}_{p}^{*}$. For a given $y_{a}=g^{X_{a}} \bmod p$ and $y_{b}=g^{X_{b}} \bmod p$, where $X_{a}$ and $X_{b}$ are randomly chosen from $\mathbb{Z}_{p}^{*}$, the following two tuples of random variables $\left(y_{a}, y_{b}, g^{X_{a} X_{b}} \bmod p\right)$ and $\left(y_{a}, y_{b}, W\right)$, where $W$ is a random value in $\mathbb{Z}_{p}^{*}$, are computationally indistinguishable. In other words, there is no efficient adversary algorithm $E$ that satisfies $\mid \operatorname{Pr}\left[E\left(g^{X_{a}}, g^{X_{b}}\right.\right.$, $\left.\left.g^{X_{a} X_{b}}\right)=\operatorname{true}\right]-\operatorname{Pr}\left[E\left(g^{X_{a}}, g^{X_{b}}, R\right)\right]>(1 / Q(|q|))$ for any polynomial $Q$, where the probability is higher than the random choice of $X_{a}, X_{b}$, and $R$.

In the proxy- and certificate-based batch authentication protocols, messages are encrypted by the ElGamal proxy cryptography. Based on Assumption 1, [23] has proven that the security of the ElGamal encryption is as hard as the DDH problem. Here, we show how we can reduce our protocols to the ElGamal encryption.

Theorem 5.1: Under Assumption 1, the proposed protocol is secure against passive adversaries.

Proof: In a proof by contradiction, assume that there is an adversary $E$ who can derive the message $\xi$ that was encrypted by the ElGamal encryption $\left(Z 1=\alpha^{r}=g^{r}, Z 2=\right.$ $\left.\xi \beta^{r}=\xi\left(\left(g^{x}\right)^{r}\right) \bmod p\right)$.
We construct another efficient algorithm $E^{\prime}$ to obtain the message $\xi$ that was encrypted by the ElGamal proxy encryption. The adversary can perform the Execute query mentioned in Section V-B-1 to obtain $\left(C 1, C 2_{R}\right),\left(C 1, C 2_{A}\right)$, and $\left(C 1, C 2_{i}\right)$ for $1 \leq i \leq|\hat{U}|$ as the inputs of the algorithm $E^{\prime}$. Without loss of generality, let $C 1=Z 1=g^{t}$ and $C 2_{R}=$ $\xi\left(g^{V_{R}}\right)^{t}$, where $t, V_{R}, N_{R}$, and $N_{A}, V_{1}, \ldots, V_{n-1}$ are random numbers that were chosen from $\mathrm{Z}_{q}^{*}$. We compute the following values:

$$
\begin{aligned}
C 2_{A}= & C 2_{R} \times C 1^{N_{R}} \\
= & \xi\left(g^{V_{R}}\right)^{t} \times C 1^{N_{R}} \\
= & \xi g^{t\left(N_{R}+V_{R}\right)} \\
C 2_{1}= & C 2_{A} \times C 1^{V_{1}} \\
= & \xi g^{\left(N_{R}+V_{R}\right) t} \times C 1^{V_{1}} \\
= & \xi g^{t\left(N_{R}+V_{R}+V_{1}\right)} \\
& \vdots \\
C 2_{|\hat{U}|}= & \left.C 2_{|\hat{U}|-1} \times C 1^{V_{|\hat{U}|}}\right) \times C 1^{V_{|\hat{U}|}} \\
= & \xi\left({ }_{j}^{|\hat{U}|-1}\right) \\
& \left.\left(N_{R}+V_{R}+\sum_{j=1}^{V_{j}}\right) t\right) \\
= & \xi g\left(N_{R}+V_{R}+\sum_{j=1}^{|\hat{U}|} V_{j}\right) t \\
= & \xi \beta^{r}=Z 2 .
\end{aligned}
$$

Now, the algorithm $E^{\prime}$ has been constructed. Because $E$ can derive the messages encrypted by the ElGamal encryption, by applying $E, E^{\prime}$ can obtain the message $\xi$ that was encrypted by the ElGamal proxy encryption, which is a contradiction to Assumption 1.

Lemma 5.2: Under Assumption 1, any adversary $E$ cannot generate a valid $C 2_{i}=\xi g^{r\left(K_{R A}+N_{R}+\sum_{j=1}^{|\hat{U}|} K_{A j}\right)} \bmod p$, which can successfully be verified by the requester $U_{R}$.

Proof: In our protocol, each user $U_{i}$ generates $C 2_{i}$ from $C 2_{i-1}$. The message $\left(C 1, C 2_{i}, X\right)$ is broadcast, where $C 1=$ $g^{r} \bmod p, C 2_{i}=\xi g^{r\left(N_{R}+K_{R A}+K_{A 1}+\cdots+K_{A i}\right)} \bmod p$, and the adversary $E$ must compute $C 2_{i}$ from these public parameters. Because the Advanced Encryption Standard (AES) algorithm is assumed to be secure [24], the parameter $X$ is well protected. Obviously, the malicious adversary $E$ must efficiently distinguish $\left(g^{r}, \xi g^{r\left(N_{R}\right)}, \xi g^{r\left(K_{R A}+N_{R}+K_{A 1}+\cdots+K_{A i}\right)} \bmod p\right)$ from $\left(g^{r}, \xi g^{r\left(N_{R}\right)}, W \bmod p\right)$, where $W$ is a random value in $\mathbb{Z}_{q}^{*}$. The problem is obviously a contradiction to Assumption 1. We then prove that no adversary $E$ can pass the authentication under Assumption 1.

Lemma 5.3: Assume that, by computing the discrete logarithms modulo a large prime is difficult, any malicious adversary $E$ cannot generate a signature protocol $\delta=$ $\operatorname{Sign}\left(R K_{R}, C 1\right)$.

Proof: Because the signature $\delta=\operatorname{Sign}\left(R K_{R}, C 1\right)$ is generated by the Shamir-Tauman online/offline signature protocol, the security proof can directly refer to [16]. We briefly quote the conclusion. If the adversary $E$, without knowing 
the secret key $R K_{R}$, can impersonate $U_{R}$ to generate valid signatures with a nonnegligible probability $\epsilon$, then the adversary $E$ can efficiently compute the discrete logarithms modulo a large prime. Thus, the adversary $E$ who tries to generate $\delta=$ $\operatorname{Sign}\left(R K_{R}, C 1\right)$, indeed, needs the secret key $R K_{R}$. In conclusion, each user $U_{i}$ can correctly authenticate the requester $U_{R}$ by signature $\delta=\operatorname{Sign}\left(R K_{R}, C 1\right)$ under the assumption of discrete logarithms.

Theorem 5.4: Under the discrete logarithm assumption and Assumption 1, the proposed proxy- and certificate-based protocols are secure against impersonator attacks.

Proof: In this proof, we first show that the requester $U_{R}$ can authenticate each user $U_{i}$ under the Diffie-Hellman problem (DH) assumption. Then, each $U_{i}$ can also authenticate $U_{R}$ under the DDH problem assumption or the discrete logarithm problem (DLP) assumption [19].

By Lemma 5.2, we know that only legal requester $U_{R}$ and users $U_{i}$, who possess the secret shared key $K_{A} i$ with the authenticator $U_{A}$, can generate $C 2^{\prime}$ and $C 2_{i}$. Therefore, the protocols based on the ElGamal proxy encryption are secure against impersonator attacks.

By Lemma 5.3, it is clear that only legal requester $U_{R}$, who holds $R K_{R}$, can generate the signature $\delta=\operatorname{Sign}\left(R K_{R}, C 1\right)$. Because the adversary has no access to the legal requester's secret key $R K_{R}$, he/she cannot generate a valid signature $\delta$. Thus, we prove that the proposed proxy- and certificate-based protocols are secure against impersonator attacks and provide mutual authentication.

Theorem 5.5: Under the discrete logarithm assumption and Assumption 1, the proposed proxy- and certificate-based protocols can provide implicit key authentication.

Proof: By Lemma 5.2, we know that any malicious adversary cannot generate $C 2_{R}$ and use it to derive the key agreement parameter $g^{m_{i}}$ under the assumption of DLP. Although the counterpart of key agreement parameter $g^{n_{i}}$ is transferred in plaintext, $M A C_{i}$ can be used to withstand the malicious adversary under the security of AES [24] and the ElGamal proxy encryption. Because " $N_{A}+N_{R}+K_{R A}+\sum_{j=1}^{|\hat{U}|} K_{A j}$ " and "S" are well protected by AES and the ElGamal proxy encryption, these values can be used to generate a valid $M A C_{i}$. Hence, the proposed proxy- and certificate-based protocols, taking the ElGamal proxy encryption as their basis, are proved to offer the implicit key authentication.

Theorem 5.6: Under the discrete logarithm assumption and Assumption 1, the proposed protocols provide forward secrecy.

Proof: In this proof, we first prove that the proxy- and certificate-based protocols guarantee forward secrecy and then show that the hash-based protocol also provides forward secrecy as follows.

- The key agreement handshakes between $U_{R}$ and $U_{i}$ are independent of the secret key $K_{R A}$ and $K_{A i}$. Without loss of generality, let $U_{p}=\left\{U_{1}, U_{2}, \ldots, U_{n}\right\}$ be the set of users who have established a session key $S K_{R p}$ with $U_{R}$ at some past time $\tau$. After closing the session, the session key $S K_{R p}$ will be tossed. Suppose that an adversary $E$ obtains a secret key $K_{A p}$ of any user $U_{p}$ and the secret key $S K_{R A}$ of $U_{R}$ at time $\tau+1$. Because the session key $S K_{R p}$ is isolated from the secret key $K_{A p}$, the adversary $E$ cannot obtain the session key $S K_{R p}$. In the case that the adversary $E$ obtains $g^{m_{p}}$ or $g^{n_{p}}$, he/she can compute the session key $S K_{R p}$. By Lemma 5.2, we know that the adversary $E$ cannot obtain $m_{p}$ or $n_{p}$ from $g^{m_{p}}$ or $g^{n_{p}}$ under the assumption of discrete logarithms. We hence prove that our protocols guarantee forward secrecy.

- In the hash-based batch authentication protocol, we exploit the DH key agreement to negotiate the session key. Therefore, its security of forward secrecy is the same as previously mentioned.

3) Security Proof by Random Oracle Concept: According to the concept of the random-oracle model [18], which assumes that the one-way hash function is a fair random function, we can prove that the proposed hash-based batch authentication protocol is secure against passive adversaries, impersonators, and implicit key authentication attacks.

Theorem 5.7: Under the assumption of the discrete logarithm and the robust one-way hash function, the proposed hashbased protocol is secure against passive adversaries.

Proof: If the adversary cannot acquire any critical meaning information from the messages transmitted in the public channel, the proposed hash-based protocol is secure against passive adversaries. In the hash-based batch authentication protocol, most messages are operated by exclusive ORs (XORs) and the one-way hash function as follows.

- Because the XOR operation is widely adapted to several famous systems [21], [22] to mix with messages that infer that the XOR operation is suited to hide information, we also apply XORs to conceal the information.

- The most important information $h_{i}$ is protected by the one-way hash function. Under the concept of the randomoracle model [18], the probability is negligible when the adversary tries to extract $h_{i}$ from $M_{i+2}=H\left(\left(N_{R}+\right.\right.$ $\left.\left.N_{A}\right) \oplus h_{i}\right)$. For $M A C_{i}$, the security of the message authentication code is also under the concept of the randomoracle model due to the indistinguishability of the one-way hash function.

Although the parameters of key agreement $g^{n_{i}}$ are exposed to the public, the assumption of a discrete logarithm ensures that the adversary does not have the nonnegligible advantage to know the value of $n_{i}$. As a result, the hash-based batch authentication protocol is secure against a passive attack.

Theorem 5.8: As long as the one-way hash function is robust, the proposed hash-based batch authentication protocol is secure against impersonator attacks.

Proof: An impersonator attack is an attack where the adversary acts on behalf of other users to pass the authentication. To impersonate a legal user, the adversary needs to obtain $h_{i}, N_{R}$, and $N_{A}$. Under the concept of the randomoracle model, which assumes that the one-way hash function is robust, the probability that the adversary obtains $h_{i}, N_{R}$, and $N_{A}$ from $M_{i+2}$ is negligible. The adversary may extract $\left(h_{i-2}, h\left(K_{A i}\right), \sum_{j=1}^{2} N_{j}, g^{m_{i}}\right)$ if he/she knows the preshared keys $K_{A i}$ and $t_{i}$ between $U_{i}$ and $U_{A}$. Because shared key $K_{A i}$ is well protected by the one-way hash function, under the concept 
TABLE II

PERFormanCE COMPARISONS OF THE UNDERLYING CRYPTOGRAPHIES

\begin{tabular}{|l||c|c|c|c|c|}
\hline \multirow{2}{*}{} & \multirow{2}{*}{ Kerberos } & \multirow{2}{*}{ ElGamal } & \multicolumn{2}{c|}{ Proposed batch authentication protocols } \\
\cline { 3 - 6 } & & & Hash- & Proxy- & Certificate- \\
\hline Communication cost & $6 n$ & $n^{2}-n$ & $2 n+2$ & $2 n+2$ & $n+3$ \\
\hline Computational cost & Low & Medium & Extremely low & Medium & High \\
\hline Underlying cryptosystem & Symmetric & Asymmetric & One-way hash & Asymmetric & Asymmetric \& PKI \\
\hline Trustworthiness & In Pairs $(U \& S)$ & In Pairs $\left(U_{R} \& U_{j}\right)$ & In Pairs $\left(U_{R} \& U_{i}\right)$ & All Entities & All Entities \\
\hline Trust level & N/A & N/A & N/A & Supported & Supported \\
\hline
\end{tabular}

$n$ : number of users to be authenticated.

of the random-oracle model, the probability of compromising the shared key from the transmitted messages is negligible. Thus, our hash-based protocol is secure against impersonator attacks.

Theorem 5.9: Under the assumption of a discrete logarithm and the robust one-way hash function, the proposed hash-based protocol is secure against implicit key authentication.

Proof: In the hash-based protocol, two parameters of key agreement are exchanged during handshake as follows.

- $g^{n_{i}}$ is transmitted in plaintext, but its integrity can be guaranteed by $M A C_{i}$.

- $g^{m_{i}}$ is protected by $H\left(r,\left(K_{A i} \| t_{i}\right)\right)$. Under the concept of the random-oracle model, only the legal $U_{i}$ who possesses the shared key $K_{A i}$ can obtain the $g^{m_{i}}$. Based on the discrete logarithm assumption, no one can obtain the value of $n_{i}$, except for the legal $U_{i}$.

Therefore, the implicit key authentication is provided in our hash-based protocol.

\section{COMPARISONS}

In this section, we compare our protocols with the Kerberos, ElGamal, and other existing authentication protocols. Because our architecture is similar to Kerberos [25], [26], we first compare our protocols with Kerberos when authenticating multiple users. Table II compares the proposed protocols with Kerberos in terms of the communication cost, computational cost, the underlying cryptosystems, trustworthiness relationship, and the support of trust level. While authenticating $n$ users, the Kerberos protocol requires $6 n$ messages to completely authenticate all users. In contrast, the proposed protocols require at most $2 n+2$ messages. To show the advantage of our batch concept, a comparison with the traditional ElGamal encryption is also presented. While authenticating $n$ users, the total number of messages is $2 \times((n \cdot(n-1)) / 2)=n^{2}-n$. The reason is that the traditional ElGamal encryption requires two messages to achieve mutual authentication. Moreover, if $n$ users try to authenticate the other users, the number of authentication times is $((n \cdot(n-1)) / 2)$. Fig. 7 illustrates the comparison of communication cost among our framework, Kerberos, and ElGamal encryption. As shown, our scheme outperforms the other schemes. Moreover, the performance advantage increases with an increase in the number of users to be authenticated.

Because the hash-based protocol takes a one-way hash function as its underlying cryptosystem, its computational costs are extremely low. Because the proposed certificate-based batch

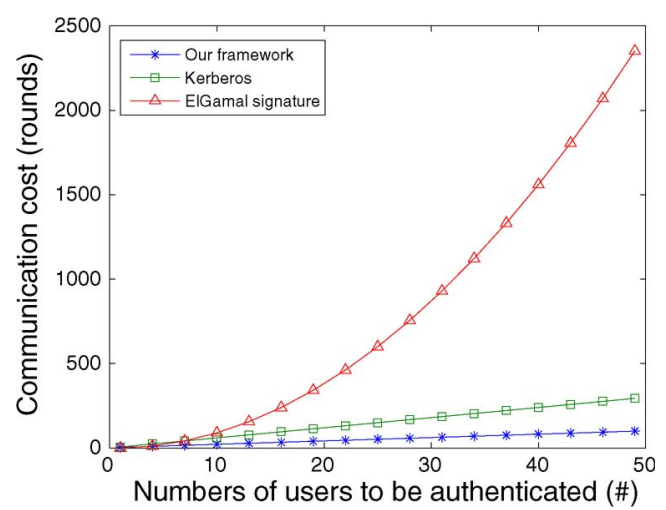

Fig. 7. Communication cost versus the number of users to be authenticated.

authentication protocol adopts the ElGamal proxy encryption scheme and public key infrastructure, its computational cost is the highest, but it requires the fewest messages for authentication. With regard to trustworthiness, both Kerberos and the proposed hash-based protocol build trustworthiness between the requester $U_{R}$ and individual user $U_{i}$. By applying the proxy- and certificate-based protocols, we can build complete trustworthiness among all entities, including $U_{A}, U_{R}$, and $U_{i} \in$ $U$. In our protocols, the trust level can be used to distinguish the level of trustworthiness. For example, a face-to-face authentication leads to a higher trust level, whereas P2P-based authentication has a lower trust level. In our protocols, only the proxy- and certificate-based protocols provide trust level during authentication. By the provided trust level, entities can predefine a trust level threshold to determine whether to accept the user as a trusted friend.

In Table III, we compare our protocols with other P2Pbased authentication protocols with respect to the requirements mentioned in Section III. Most protocols provide mutual authentication among entities, but none of the existing protocols offers batch authentication. The proposed proxy- and certificate-based protocols also support user reputation by the trust level. Moreover, the certificate-based protocol also offers nonrepudiation by signing the messages. Because no centralized server is involved in our design, the proposed protocols can be adapted to any OSN, either web- or P2P-based architecture. Our framework also supports comprehensive hardware, from resource-limited to powerful computing devices. As previously mentioned, the hash-based protocol takes only a one-way hash function as its underlying cryptosystem and, hence, is cost effective. In short, our protocols meet most of the requirements mentioned in Section III. 
TABLE III

FeAture Comparisons of the P2P-BAsed Authentication Protocols

\begin{tabular}{|c|c|c|c|c|c|c|c|c|c|c|c|c|c|}
\hline & VistualSec & Safebook $^{*}$ & Groove & FL02 & AAPR & GD03 & N06 & \multirow{2}{*}{ PGP } & \multirow{2}{*}{ Kerberos } & \multirow{2}{*}{ ElGamal } & \multicolumn{3}{|c|}{ Our Protocols } \\
\hline & [3] & [6] & [27] & [28] & [9] & [8] & [10] & & & & Hash- & Proxy- & Certificate- \\
\hline $\begin{array}{ll}\text { R1. } & \begin{array}{l}\text { Strong } \\
\text { Authen. }\end{array} \\
\end{array}$ & $\Delta$ & o & o & $\mathrm{o}$ & o & o & $\mathrm{O}$ & o & o & $\mathrm{O}$ & $\mathrm{o}$ & o & $\mathrm{O}$ \\
\hline R2. Reputation & $\mathrm{x}$ & o & $\mathrm{x}$ & o & o & o & $\mathrm{x}$ & $\Delta$ & $\mathrm{x}$ & $\mathrm{x}$ & $\mathrm{x}$ & o & o \\
\hline $\begin{array}{ll}\text { R3. } & \text { Community } \\
\text { Authenticity }\end{array}$ & $\mathrm{O}$ & $\mathrm{x}$ & $\mathrm{O}$ & $\mathrm{x}$ & o & $\mathrm{O}$ & $\mathrm{O}$ & $\mathrm{O}$ & o & $\mathrm{x}$ & o & O & $\mathrm{O}$ \\
\hline R4. Non-repud. & $\mathrm{x}$ & 0 & $\mathrm{x}$ & $\mathrm{x}$ & o & $\mathrm{x}$ & $\mathrm{x}$ & o & $\mathrm{x}$ & o & $\mathrm{x}$ & $\mathrm{x}$ & $\mathrm{O}$ \\
\hline R5. Flexibility & o & o & o & $\mathrm{x}$ & $\mathrm{x}$ & $\mathrm{o}$ & $\Delta$ & o & $\mathrm{x}$ & o & o & o & o \\
\hline $\begin{array}{l}\text { R6. Batch } \\
\text { Authen. }\end{array}$ & $\mathrm{x}$ & $\mathrm{x}$ & $\mathrm{x}$ & $\mathrm{x}$ & $\mathrm{x}$ & $\mathrm{x}$ & $\mathrm{x}$ & $\mathrm{x}$ & $\mathrm{x}$ & $\mathrm{x}$ & o & o & o \\
\hline $\begin{array}{ll}\text { Compre. } \\
\text { R7. } \\
\text { Hardware } \\
\text { Supports }\end{array}$ & $\mathrm{x}$ & $\mathrm{x}$ & $\mathrm{x}$ & $\mathrm{x}$ & $\mathrm{x}$ & $\mathrm{x}$ & o & $\mathrm{x}$ & $\mathrm{x}$ & $\mathrm{x}$ & & o & \\
\hline
\end{tabular}

O: Supported; X: Not supported; $\triangle$ : Partially supported

*: No specific protocol descriptions

\section{CONCLUSION}

In this paper, we have proposed a P2P-based batch authentication framework for OSNs. We have also designed three batch authentication protocols using the one-way hash function, ElGamal proxy encryption, and certificates for different situations and purposes. The hash-based protocol adopts lightweight cryptosystems to reduce the computational costs. To offer higher security properties, the proxy- and certificatebased methods are based on asymmetric encryptions and signature methods to fulfil the security requirements of sensitive transactions.

In this paper, we proved that our protocols are secure against passive adversaries and impersonator attacks and support implicit key authentication. After analyzing the communication and computational costs, we show that our protocols require fewer authentication messages. Notably, the proposed hash-based batch authentication protocol is cost effective when run with resource-limited devices. Compared with other existing P2P-based authentication protocols, the proposed protocols can meet the security requirements of P2P-based OSNs, including mutual authentication, reputation, community authenticity, nonrepudiation, flexibility, and comprehensive hardware support.

\section{REFERENCES}

[1] C. Zhang, J. Sun, X. Zhu, and Y. Fang, "Privacy and security for online social networks: Challenges and opportunities," IEEE Netw., vol. 24, no. 4, pp. 13-18, Jul./Aug. 2010.

[2] D. Niyato, P. Wang, W. Saad, and A. Hjorungnes, "Controlled coalitional games for cooperative mobile social networks," IEEE Trans. on Vehi. Tech., vol. 60, no. 4, pp. 1812-1824, May 2011.

[3] M. Ge, K.-Y. Lam, X. Wang, Z. Wan, and B. Jiang, "VisualSec: A secure message delivery scheme for online social networks based on profile images," in Proc. IEEE GLOBECOM, 2009, pp. 1-6.

[4] S. Buchegger and A. Datta, "A case for P2P infrastructure for social networks-Opportunities and challenges," in Proc. WONS, 2009, pp. 161-168.

[5] S. Buchegger, D. Schioberg, L. H. Vu, and A. Datta, "PeerSoN-P2P social networking: Early experiences and insights," in Proc. SocialNets, 2009, pp. 46-52.

[6] L. A. Cutillo and R. Molva, "Safebook: A privacy-preserving online social network leveraging on real-life trust," IEEE Commun. Mag., vol. 47, no. 12, pp. 94-101, Dec. 2009.

[7] U. Lee, J. Sewook, C. Dae-Ki, A. Chang, C. Junho, and M. Gerla, "P2P content distribution to mobile Bluetooth users," IEEE Trans. Veh. Technol., vol. 59, no. 1, pp. 356-367, Jan. 2010.
[8] S. Gokhale and P. Dasgupta, "Distributed authentication for peer-topeer networks," in Proc. Appl. Internet Workshops, Jan. 27-31, 2003, pp. 347-353.

[9] H. Lee and K. Kim, "An adaptive authentication protocol based on reputation for peer-to-peer system," in Proc. Symp. Crypto. Info. Sec., 2003, pp. 661-666.

[10] K. V. Nguyen, "Simplifying peer-to-peer device authentication using identity-based cryptography," in Proc. ICNS, 2006, p. 43.

[11] D. Balfanz, D. Smetters, P. Stewart, and H. Wong, "Talking to strangers: Authentication in ad hoc wireless networks," in Proc. Symp. NDSS, San Diego, CA, Feb. 2002.

[12] C.-Y. Huang, Y.-P. Chiu, K.-T. Chen, and C.-L. Lei, "Secure multicast in dynamic environments," Comput. Netw., vol. 51, no. 10, pp. 2805-2817, Jul. 2007. [Online]. Available: http://www.sciencedirect.com/science/ article/B6VRG-4MM1P6H-5/1/fca29634eb800ee4f1ef830c09d8aefd

[13] M. Cagalj, S. Capkun, and J.-P. Hubaux, "Key agreement in peer-to-peer wireless networks," Proc. IEEE, vol. 94, no. 2, pp. 467-478, Feb. 2006.

[14] A. C. Squicciarini, F. Paci, E. Bertino, A. Trombetta, and S. Braghin, "Group-based negotiations in P2P systems," IEEE Trans. Parallel Distrib. Syst., vol. 21, no. 10, pp. 1473-1486, Oct. 2010.

[15] Y.-M. Tseng, "A secure authenticated group key agreement protocol for resource-limited mobile devices," Comput. J., vol. 50, no. 1, pp. 41-52, Jan. 2007.

[16] A. Shamir and Y. Tauman, "Improved online/offline signature schemes," in Proc. Adv. CRYPTO, 2001, pp. 355-367.

[17] G.-H. Chiou and W.-T. Chen, "Secure broadcasting using the secure lock," IEEE Trans. Soft. Eng., vol. 15, no. 8, pp. 929-934, Aug. 1989.

[18] M. Bellare and P. Rogaway, "Random oracles are practical: A paradigm for designing efficient protocols," in Proc. ACM CCS, 1993, pp. 62-73.

[19] V. Shoup, "Lower bounds for discrete logarithms and related problems," in Proc. Adv. Cryptol.-EUROCRYPT, 1997, pp. 256-266.

[20] D. Boneh, "The decision Diffie-Hellman problem," in Proc. Algorithmic Number Theory, 1998, pp. 48-63.

[21] M. Bellare, R. Guerin, and P. Rogaway, "XOR MACs: New methods for message authentication using finite pseudorandom functions," in Proc. Adv. CRYPTO, 1995, pp. 15-28.

[22] R. Rivest, "The RC5 encryption algorithm," in Proc. Fast Softw. Encryption, 1995, pp. 86-96.

[23] Y. Tsiounis and M. Yung, "On the security of ElGamal-based encryption," in Proc. Public Key Cryptography, 1998, pp. 117-134.

[24] Advanced Encryption Standard (AES), F. 197, Nov. 2001

[25] B. C. Neuman and T. Ts'o, "Kerberos: An authentication service for computer networks," IEEE Commun. Mag., vol. 32, no. 9, pp. 33-38, Sep. 1994.

[26] B.-T. Oh, S.-B. Lee, and H.-J. Park, "A peer mutual authentication method using PKI on superpeer-based peer-to-peer systems," in Proc. ICACT, 2008, pp. 2221-2225.

[27] G. Networks, A White paper: Groove Security Architecture, 2002. [Online]. Available: http://www.groove.net/products/workspace/security.html

[28] D. Fahrenholtz and W. Lamersdorf, "Transactional security for a distributed reputation management system," in Proc. ECommerce and Web Technol., 2002, pp. 214-223.

[29] C.-I. Fan and Y.-H. Lin, "Provably secure remote truly three-factor authentication scheme with privacy protection on biometrics," IEEE Trans. Inf. Forensics Security, vol. 4, no. 4, pp. 933-945, Dec. 2009.

[30] C.-I. Fan, L.-Y. Huang, and P.-H. Ho, "Anonymous multireceiver identitybased encryption," IEEE Trans. Comput., vol. 59, no. 9, pp. 1239-1249, Sep. 2010. 


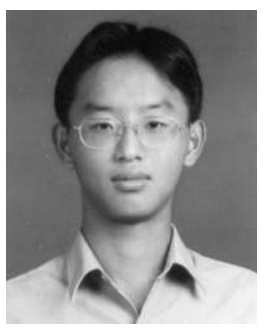

Lo-Yao Yeh (M'12) received the M.S. degree from the National Chi Nan University, Nantou, Taiwan, in 2005 and the Ph.D. degree from the National Chiao Tung University, Hsinchu, Taiwan, in 2010.

He was a Visiting Scholar with the University of California, Berkeley. He is currently an Associate Researcher with the National Center for HighPerformance Computing, Hsinchu, Taiwan, and is also an Adjunct Assistant Professor with the Department of Information Management, National Chi Nan University, Nantou, Taiwan. His research interests include cryptography, network security, vehicular networks security, and Botnet.

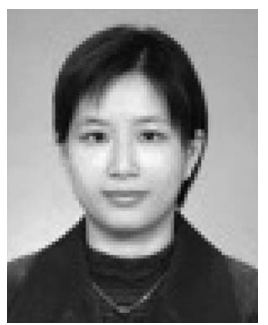

Yu-Lun Huang (M'04) received the B.S. and $\mathrm{Ph} . \mathrm{D}$. degrees in computer science and information engineering from the National Chiao-Tung University, Hsinchu, Taiwan, in 1995 and 2001, respectively.

She is currently an Assistant Professor with the Department of Electrical and Control Engineering, National Chiao-Tung University. Her research interests include wireless security, secure testbed design, embedded software, embedded operating systems, risk assessment, secure payment systems, voice over Internet protocol, quality of service, and virtualization security.

Dr. Huang is a member of the Phi Tau Phi Society.

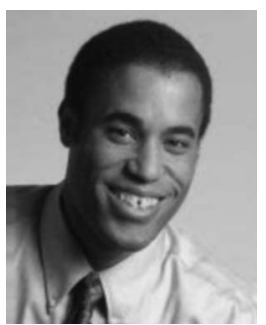

Anthony D. Joseph (M'98) received the Ph.D. degree from Massachusetts Institute of Technology (MIT), Cambridge, in 1998.

He was a Principal Designer with MIT, where he worked on the Rover mobile toolkit for providing application support for intermittent connectivity in a mobile computing environment. He is currently an Associate Professor with the Department of Electrical Engineering and Computer Sciences, University of California, Berkeley, where he is developing more accurate network modeling and emulation tools and building proxy-based computing environments for mobile code and data. His research interests include computer systems and networking, particularly mobile systems, overlay networks, code and data migration, and network security.

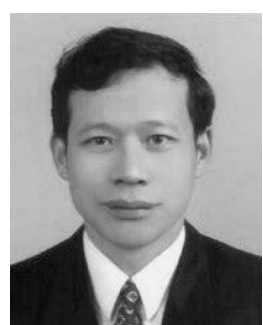

Shiuhpyng Winston Shieh (SM'98) received the M.S. and Ph.D. degrees in electrical and computer engineering from the University of Maryland, College Park.

From 2003 to 2004 and from 2005 to 2006, he was a Visiting Professor with the University of California, Berkeley. He is currently a Professor with the Department of Computer Science, National Chiao Tung University, Hsinchu, Taiwan, where he is also the Director of the Taiwan Information Security Center. His research interests include reliability and security hybrid mechanisms, network and system security, and software program behavior analysis.

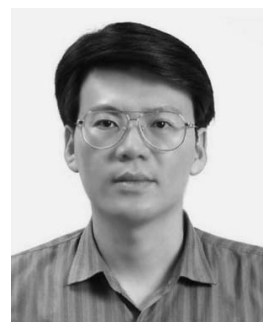

Woei-Jiunn Tsaur (M'10) received the Ph.D. degree in electrical engineering from the National Taiwan University of Science and Technology, Taipei, Taiwan, in 1998.

From 1994 to 2003, he was a Project Manager and Technology Consultant with the R\&D Division, Syscom Computer Engineering Company, Taipei, Taiwan, a research center of software development. Since 1999, he has been with the Department of Information Management, Da-Yeh University, Changhua, Taiwan, where he is currently a Full Professor. His research interests include network security, security topics in operating systems, applied cryptography, information security management, and computer networks. 